\title{
Nonlinear Finite Element Analysis of Free and Forced Vibrations of Cellular Plates Having Triangularly Prismatic Metamaterial Cores: A Strain Gradient Plate Model Approach
}

jalal Torabi ( $\sim$ Jalal.Torabi@aalto.fi )

Aalto-yliopisto https://orcid.org/0000-0001-7525-8442

Jarkko Niiranen

Aalto-yliopisto

\section{Research Article}

Keywords: Strain gradient elasticity, Nonlinear vibration, C1-continuous finite element, Microarchitecture, Mechanical metamaterials, Cellular plates

Posted Date: October 13th, 2021

DOl: https://doi.org/10.21203/rs.3.rs-964566/v1

License: (c) (1) This work is licensed under a Creative Commons Attribution 4.0 International License.

Read Full License 


\title{
Nonlinear finite element analysis of free and forced vibrations of cellular plates having triangularly prismatic metamaterial cores: a strain gradient plate model approach
}

\author{
Jalal Torabi, Jarkko Niiranen \\ Department of Civil Engineering, School of Engineering, Aalto University \\ P.O. Box 12100, Aalto 00076, Finland
}

\begin{abstract}
The main objective of this paper is to develop a theoretically and numerically reliable and efficient methodology based on combining a finite element method and a strain gradient shear deformation plate model accounting for the nonlinear free and forced vibrations of cellular plates having equitriangularly prismatic metamaterial cores. The proposed model based on the nonlinear finite element strain gradient elasticity is developed for the first time to provide a computationally efficient framework for the simulation of the underlying nonlinear dynamics of cellular plates with advanced microarchitectures. The corresponding governing equations follow Mindlin's SG elasticity theory including the micro-inertia effect applied to the first-order shear deformation plate theory along with the nonlinear von Kármán kinematics. Standard and higher-order computational homogenization methods determine the classical and strain gradient material constants, respectively. A higher-order $C^{1}$-continuous 6-node finite element is adopted for the discretization of the governing variational formulation with respect to the spatial domain, and an arc-length continuation technique along with time periodic discretization is implemented to solve the resulting nonlinear time-dependent problem. Through a set of comparative studies with 3D full-field models as references, the accuracy and efficacy of the proposed dimension reduction methodology are demonstrated for a diverse range of problem parameters for analyzing the large-amplitude dynamic structural response.
\end{abstract}

Keywords: Strain gradient elasticity; Nonlinear vibration; $C^{1}$-continuous finite element; Microarchitecture; Mechanical metamaterials; Cellular plates

\section{Introduction}

The rapid development of manufacturing technologies, in the field of additive manufacturing processes particularly, expands the spaces of production procedures and applications of advanced mechanical metamaterials and microarchitectural structures in various engineering fields [1]. According to these developments and the great fundamental characteristics of lattice architectures such as adjustable 
stiffness-to-weigh ratio and high surface-to-volume ratio, there are essential demands for developing reliable and efficient computational methodologies to predict the thermomechanical characteristics of these state-of-the-art artifacts.

The structural models based on the classical continuum mechanics have been effectively utilized to simulate the static and dynamic behavior of various engineering structures already for decades if not centuries. However, the complex internal microarchitectures of advanced cellular structures and the high computational costs of the common numerical approaches such as the conventional finite element methods, when adopted in the form of detailed modeling, considerably reduce the applicability of classical models regarding the structural simulation of mechanical metamaterials. In contrast, the generalized continuum theories like the microcontinuum theory [2], strain gradient theory (SGT) [3,4], and nonlocal theory [5] propose higher-order (or higher-grade) models by which the complexity of the microarchitecture-dependent behavior of cellular or lattice structures or the microstructure-dependent mechanics of micro/nanostructures can be efficiently handled. Indeed, the application of generalized continuum theories in the field of mechanics can be considered from two different viewpoints. First, by following experimental observations [6,7] on the size-dependent behavior of structures at micro/nano scales, these higher-order models have been extensively applied to capture this size-effect [8-14]. In the meantime, several studies on the linear and nonlinear structural models of beams [Error! Reference source not found.-17], plates [18-Error! Reference source not found.], and shells [23] within the SGT have been reported in the literature. Besides, different numerical solution methods such as higher-order finite element methods [24-29], and isogeometric analysis [30-33] have been developed. Second, the generalized continuum theories can be usefully employed to consider the microarchitecture-dependent mechanics of lattice or cellular structures [34-40]. Indeed, by using these higher-order theories, the internal microarchitecture can be homogenized via generalized constitutive relations, possibly together with dimension reduction models, which remarkably decreases both modeling and computation costs.

A literature survey discloses a rather limited number of studies on the mechanics of cellular materials under the higher-order continuum theories [34-40]. Since solids with different microarchitectures under various loading conditions and deformation states might not be appropriately modeled by using a unified generalized continuum theory, different models such as the Cosserat theory [41], micropolar theory $[35,42,43]$ and higher gradient theories [34,37-40,44,45,46-48] have been developed. Closely connected to the present study, micropolar plate models were adopted in $[42,43]$ to present a $2 \mathrm{D}$ equivalent singlelayer for lattice core sandwich plates and panels, and the resulting problem was solved by linear Lagrange interpolation finite elements along with the selective reduced integration approach. SGT-based theoretical studies on the structural analysis and the determination of higher-order constitutive parameters of cellular 
beams and plates with prismatic cores have been reported by Khakalo et al. in [46-48], with the corresponding kinematic formulations for both thin and thick structures and with isogeometric analysis for numerical solutions. Besides, the nonlinear static bending of cellular plates with an equitriangular microarchitecture was recently studied by Torabi and Niiranen in [49] highlighting the importance of geometric nonlinearity. Analytical studies on pantographic structures within a higher gradient theory with numerically and experimentally validated results can be found in $[44,45]$. Numerical homogenization within the SGT for a two-dimensional lattice structure was presented by Auffray et al. [38].

There exists only a few contributions on the dynamics of cellular structures within generalized continuum theories: the wave propagation analysis in [50,51]; the investigations of linear vibrations based on the micropolar theory [43,52,53], couple-stress theory [54], nonlocal theory [55] and SGT [46]. However, the effects of geometrical nonlinearities on the vibrations of cellular structures within the generalized continuum theories have not been studied. Accordingly, inspired by the work of Khakalo and Niiranen [48] in which the classical and SG constitutive parameters of SG plate models have been determined via numerical homogenization techniques, this study investigates by adopting a higher-order finite element (FE) method the geometrically nonlinear free/forced vibrations of cellular plates having triangularly prismatic cores formed by extruding $2 \mathrm{D}$ equitriangular lattices in the third coordinate direction.

This study formulates the problems of structural dynamics by following Mindlin's anisotropic SGT (Section 1) along with the first-order shear deformation plate theory (FSDPT) and von Kármán's hypothesis (Section 2). The bending-related classical and SG constitutive parameters from [48] are extended to the nonlinear formulation according to [49]. To facilitate the implementation of a higherorder FE method, a matrix form Hamiltonian of the problem set is first derived (Section 4), whereas the corresponding FE formulation (Section 5) is obtained by relying on a quasi-conforming 6-node triangular element (Section 6), to appropriately discretize the variational formulation with respect to the spatial domain. Time integration and arc-length continuation are finally employed for solving the nonlinear timedependent problem of forced vibration responses. Finally (Section 7), the reliability of the FE model is confirmed by convergence studies for free vibrations. Then a set of comparison studies between the SG plate model and the related three-dimensional full-field one are performed for linear natural frequencies to ensure the accuracy of the plate model further in nonlinear vibrations. These comparisons are accomplished for different plate geometries and parameter ranges. Microarchitecture effects as well as force amplitude and damping coefficient are in the focus of the studies on the nonlinear vibration responses. 


\section{Anisotropic strain gradient theory}

On the basis of the fundamentals of the SG theory proposed by Mindlin [19], the strain energy of solids (with the occupied volume of $\mathcal{V}$ ) is defined by using strain and first strain gradient tensors $\left(\epsilon_{i j}, \eta_{i j k}\right)$, and corresponding classical and higher-order stress tensors $\left(\sigma_{i j}, \Sigma_{i j k}\right)$ as

$\Pi=\int_{\mathcal{V}}\left(\sigma_{i j} \epsilon_{i j}+\eta_{i j k} \Sigma_{i j k}\right) d \mathcal{V}$

where the kinematic and constitutive relations for the strain and classical Cauchy stress tensors are expressed by

$\epsilon_{i j}=\frac{1}{2}\left(U_{i, j}+U_{j, i}+U_{k, i} U_{k, j}\right)=\epsilon_{j i}$,

$\sigma_{i j}=C_{i j k l} \epsilon_{k l}$

with $U_{i}$ as the components of the displacement and $C_{i j k l}$ as the classical material stiffness tensor. In addition, the first strain gradient tensor and associated higher-order stress tensor are presented as

$\eta_{i j k}=\epsilon_{i j, k}=\eta_{j i k}$

$\Sigma_{i j k}=A_{i j k l m n} \eta_{l m n}$

with $A_{i j k l m n}$ as the sixth-order stiffness tensor related to the SG theory. On the other hand, by considering $d_{i j}$ as the inertial length scale parameters, the kinematic energy is expressed as

$\mathcal{T}=\int_{\mathcal{V}} \frac{1}{2}\left(\rho \dot{U}_{i} \dot{U}_{i}+\rho d_{i j} \dot{U}_{i, k} \dot{U}_{j, k}\right) d \mathcal{V}$

Various studies with more detailed explanations of Mindlin's SG elasticity theory have been published in the open literature and more interested readers can refer to [3,18,Error! Reference source not found.,26,48]. Herein, the geometrically nonlinear shear deformable plate model in conjunction with von Kármán's kinematic relations is detailedly defined within the SG elasticity theory in the next section.

\section{Nonlinear shear deformation plate model within SG theory}

Following the FSDPT and SG elasticity model, this section represents the kinematic and constitutive relations and defines the matrix-vector form of Hamilton's principle. To this end, first, the Cartesian coordinate system of $\mathbf{x}=\left(x_{1}, x_{2}, x_{3}\right)$ is considered and by introducing $u_{1}, u_{2}, u_{3}$ as the mid-plane displacements and $\psi_{1}, \psi_{2}$ as the mid-plane rotations, the displacement field of the plate is presented as follows:

$U_{1}(\mathbf{x}, t)=u_{1}+x_{3} \psi_{1}$,

$U_{2}(\mathbf{x}, t)=u_{2}+x_{3} \psi_{2}$,

$U_{3}(\mathbf{x}, t)=u_{3}$ 
in which $u_{1}, u_{2}, u_{3}, \psi_{1}, \psi_{2}$ are functions of $x_{1}, x_{2}, t$ where $t$ stands for time. The displacement field of Eq. (5) can be rewritten in the form

$\mathbf{U}=\left[\begin{array}{l}U_{1} \\ U_{2} \\ U_{3}\end{array}\right]=\mathbf{A}_{0} \mathbf{u}, \quad \mathbf{A}_{0}=\left[\begin{array}{ccccc}1 & 0 & 0 & x_{3} & 0 \\ 0 & 1 & 0 & 0 & x_{3} \\ 0 & 0 & 1 & 0 & 0\end{array}\right], \quad \mathbf{u}=\left[\begin{array}{l}u_{1} \\ u_{2} \\ u_{3} \\ \psi_{1} \\ \psi_{2}\end{array}\right]$.

In accordance with the displacement field and by following von Karman's nonlinear kinematic relations, the strain vector is presented as

$\boldsymbol{\epsilon}=\left\{\begin{array}{l}\epsilon_{11} \\ \epsilon_{22} \\ \gamma_{12} \\ \gamma_{13} \\ \gamma_{23}\end{array}\right\}=\left\{\begin{array}{c}u_{1,1}+x_{3} \psi_{1,1} \\ u_{2,2}+x_{3} \psi_{2,2} \\ u_{1,2}+u_{2,1}+x_{3}\left(\psi_{1,2}+\psi_{2,1}\right) \\ \psi_{1}+u_{3,1} \\ \psi_{2}+u_{3,2}\end{array}\right\}+\frac{1}{2}\left\{\begin{array}{c}u_{3,1}^{2} \\ u_{3,2}^{2} \\ 2 u_{3,1} u_{3,2} \\ 0 \\ 0\end{array}\right\}$.

We note that for instance $u_{1,1}$ is the first-order derivative of $u_{1}$ to coordinate $x_{1}$. With the aid of introduced strain components and by following the first relation of Eq. (3) for the strain gradient tensor (i.e., $\eta_{i j k}=\epsilon_{i j, k}$ ), the strain gradient vector can be defined as

$$
\begin{aligned}
& \boldsymbol{\eta}=\left\{\begin{array}{l}
\boldsymbol{\eta}_{1} \\
\boldsymbol{\eta}_{2} \\
\boldsymbol{\eta}_{3} \\
\boldsymbol{\eta}_{4}
\end{array}\right\}, \\
& \boldsymbol{\eta}_{1}=\left\{\begin{array}{l}
\eta_{111} \\
\eta_{221} \\
\eta_{212}
\end{array}\right\}=\left\{\begin{array}{c}
u_{1,11}+x_{3} \psi_{1,11} \\
u_{2,12}+x_{3} \psi_{2,12} \\
u_{1,22}+u_{2,12}+x_{3}\left(\psi_{1,22}+\psi_{2,12}\right)
\end{array}\right\}+\left\{\begin{array}{c}
u_{3,1} u_{3,11} \\
u_{3,2} u_{3,12} \\
u_{3,1} u_{3,22}+u_{3,2} u_{3,12}
\end{array}\right\}, \\
& \boldsymbol{\eta}_{2}=\left\{\begin{array}{c}
u_{2,22}+x_{3} \psi_{2,22} \\
u_{1,12}+x_{3} \psi_{1,12} \\
\eta_{112} \\
\eta_{121}
\end{array}\right\}=\left\{\begin{array}{c}
u_{3,2} u_{3,22} \\
u_{3,1} u_{3,12} \\
u_{1,12}+u_{2,11}+x_{3}\left(\psi_{1,12}+\psi_{2,11}\right)
\end{array}\right\}+\left\{\begin{array}{c}
u_{3,2} \\
u_{3,11}+u_{3,1} u_{3,12}
\end{array}\right\}, \\
& \boldsymbol{\eta}_{3}=\left\{\begin{array}{l}
\boldsymbol{\eta}_{1,1} \\
\eta_{113} \\
\eta_{131} \\
\eta_{223} \\
\eta_{232}
\end{array}\right\}=\left\{\begin{array}{c}
\eta_{123} \\
\psi_{1,1}+u_{3,11} \\
\psi_{2,2} \\
\eta_{231}+u_{3,22} \\
\eta_{312}
\end{array}\right\}=\left\{\begin{array}{l}
\psi_{1,2}+\psi_{2,1} \\
\psi_{2,1}+u_{3,12} \\
\psi_{1,2}+u_{3,12}
\end{array}\right\},
\end{aligned}
$$

To make the governing equations more appropriate for a finite element study, the matrix forms of strain and strain gradient vectors are introduced by the following relations:

$\boldsymbol{\epsilon}=\left(\mathbf{A E}+\frac{1}{2} \mathbf{A}^{n} \mathbf{E}^{n}\right) \mathbf{u}$,

$\boldsymbol{\kappa}_{1}=\left(\mathbf{A}_{1} \mathbf{E}_{1}+\frac{1}{2} \mathbf{A}_{1}^{n} \mathbf{E}_{1}^{n}\right) \mathbf{u}, \quad \boldsymbol{\kappa}_{2}=\left(\mathbf{A}_{2} \mathbf{E}_{2}+\frac{1}{2} \mathbf{A}_{2}^{n} \mathbf{E}_{2}^{n}\right) \mathbf{u}, \quad \boldsymbol{\kappa}_{3}=\mathbf{E}_{3} \mathbf{u}, \quad \boldsymbol{\kappa}_{4}=\mathbf{E}_{4} \mathbf{u}$,

where $\mathbf{u}=\left[\begin{array}{lllll}u_{1} & u_{2} & u_{3} & \psi_{1} & \psi_{2}\end{array}\right]^{\mathrm{T}}$ gives the displacement vector and the linear classical (E) and strain gradient $\left(\mathbf{E}_{i}, i=1,2,3,4\right)$ matrix operators can be defined based on Eqs. (7) and (9-11), respectively, as follows: 
$\mathbf{E}=\left[\begin{array}{ccccc}\partial_{1} & 0 & 0 & 0 & 0 \\ 0 & \partial_{2} & 0 & 0 & 0 \\ \partial_{2} & \partial_{1} & 0 & 0 & 0 \\ 0 & 0 & 0 & \partial_{1} & 0 \\ 0 & 0 & 0 & 0 & \partial_{2} \\ 0 & 0 & 0 & \partial_{2} & \partial_{1} \\ 0 & 0 & \partial_{1} & 1 & 0 \\ 0 & 0 & \partial_{2} & 0 & 1\end{array}\right]$,

$\mathbf{E}_{1}=\left[\begin{array}{ccccc}\partial_{11} & 0 & 0 & 0 & 0 \\ 0 & \partial_{12} & 0 & 0 & 0 \\ \partial_{22} & \partial_{12} & 0 & 0 & 0 \\ 0 & 0 & \partial_{11} & 0 & 0 \\ 0 & 0 & 0 & 0 & \partial_{12} \\ 0 & 0 & \partial_{22} & 0 & \partial_{12}\end{array}\right], \quad \mathbf{E}_{2}=\left[\begin{array}{ccccc}0 & \partial_{22} & 0 & 0 & 0 \\ \partial_{12} & 0 & 0 & 0 & 0 \\ \partial_{12} & \partial_{22} & 0 & 0 & 0 \\ 0 & 0 & 0 & 0 & \partial_{22} \\ 0 & 0 & 0 & \partial_{12} & 0 \\ 0 & 0 & 0 & \partial_{12} & \partial_{11}\end{array}\right]$,

$\mathbf{E}_{3}=\left[\begin{array}{ccccc}0 & 0 & 0 & \partial_{1} & 0 \\ 0 & 0 & \partial_{11} & \partial_{1} & 0 \\ 0 & 0 & 0 & 0 & \partial_{2} \\ 0 & 0 & \partial_{22} & 0 & \partial_{2}\end{array}\right], \quad \mathbf{E}_{4}=\left[\begin{array}{ccccc}0 & 0 & 0 & \partial_{2} & \partial_{1} \\ 0 & 0 & \partial_{12} & 0 & \partial_{1} \\ 0 & 0 & \partial_{12} & \partial_{2} & 0\end{array}\right]$,

where $\partial_{1}=\partial / \partial x_{1}, \partial_{2}=\partial / \partial x_{2}, \partial_{11}=\partial^{2} / \partial x_{1}^{2}, \partial_{22}=\partial^{2} / \partial x_{2}^{2}, \partial_{12}=\partial^{2} / \partial x_{1} \partial x_{2} . \quad$ Besides, the nonlinear classical $\left(\mathbf{E}^{n}\right)$ and strain gradient $\left(\mathbf{E}_{i}^{n}, i=1,2\right)$ matrix operators are expressed as

$\mathbf{E}^{n}=\langle\mathbf{Q} \mathbf{u}\rangle \overline{\mathbf{Q}}+\langle\overline{\mathbf{Q}} \mathbf{u}\rangle \mathbf{Q}$,

$\mathbf{E}_{1}^{n}=\left\langle\mathbf{Q}_{1} \mathbf{d}\right\rangle \overline{\mathbf{Q}}_{1}+\left\langle\overline{\mathbf{Q}}_{1} \mathbf{d}\right\rangle \mathbf{Q}_{1}, \quad \mathbf{E}_{2}^{n}=\left\langle\mathbf{Q}_{2} \mathbf{d}\right\rangle \overline{\mathbf{Q}}_{2}+\left\langle\overline{\mathbf{Q}}_{2} \mathbf{d}\right\rangle \mathbf{Q}_{2}$,

in which

$\mathbf{Q}=\left[\begin{array}{lllll}\mathbf{0} & \mathbf{0} & \mathbf{L} & \mathbf{0} & \mathbf{0}\end{array}\right], \quad \overline{\mathbf{Q}}=\left[\begin{array}{lllll}\mathbf{0} & \mathbf{0} & \overline{\mathbf{L}} & \mathbf{0} & \mathbf{0}\end{array}\right]$,

$\mathbf{Q}_{1}=\left[\begin{array}{lllll}\mathbf{0} & \mathbf{0} & \mathbf{L}_{1} & \mathbf{0} & \mathbf{0}\end{array}\right], \quad \overline{\mathbf{Q}}_{1}=\left[\begin{array}{lllll}\mathbf{0} & \mathbf{0} & \overline{\mathbf{L}}_{1} & \mathbf{0} & \mathbf{0}\end{array}\right]$,

$\mathbf{Q}_{2}=\left[\begin{array}{lllll}\mathbf{0} & \mathbf{0} & \mathbf{L}_{2} & \mathbf{0} & \mathbf{0}\end{array}\right], \quad \overline{\mathbf{Q}}_{2}=\left[\begin{array}{lllll}\mathbf{0} & \mathbf{0} & \overline{\mathbf{L}}_{2} & \mathbf{0} & \mathbf{0}\end{array}\right]$,

with

$\mathbf{L}=\left[\begin{array}{l}\partial_{1} \\ \partial_{2} \\ \partial_{1}\end{array}\right], \quad \overline{\mathbf{L}}=\frac{1}{2}\left[\begin{array}{c}\partial_{1} \\ \partial_{2} \\ 2 \partial_{2}\end{array}\right]$

$\mathbf{L}_{1}=\left[\begin{array}{l}\partial_{1} \\ \partial_{2} \\ \partial_{1} \\ \partial_{2}\end{array}\right], \overline{\mathbf{L}}_{1}=\left[\begin{array}{l}\partial_{11} \\ \partial_{12} \\ \partial_{22} \\ \partial_{12}\end{array}\right], \mathbf{L}_{2}=\left[\begin{array}{l}\partial_{2} \\ \partial_{1} \\ \partial_{2} \\ \partial_{1}\end{array}\right], \overline{\mathbf{L}}_{2}=\left[\begin{array}{l}\partial_{22} \\ \partial_{12} \\ \partial_{11} \\ \partial_{12}\end{array}\right]$

Furthermore, the matrices $\mathbf{A}, \mathbf{A}^{n}$ and $\mathbf{A}_{i}, \mathbf{A}_{i}^{n}(\mathrm{i}=1,2)$ are given by the following relations:

$\mathbf{A}=\left[\begin{array}{ccc}\mathbf{I}_{33} & x_{3} \mathbf{I}_{33} & \mathbf{0} \\ \mathbf{0} & \mathbf{0} & \mathbf{I}_{22}\end{array}\right], \quad \mathbf{A}^{n}=\left[\begin{array}{l}\mathbf{I}_{33} \\ \mathbf{0}_{22}\end{array}\right]$

$\mathbf{A}_{1}=\left[\begin{array}{ll}\mathbf{I}_{33} & x_{3} \mathbf{I}_{33}\end{array}\right], \quad \mathbf{A}_{1}^{n}=\left[\begin{array}{llll}1 & 0 & 0 & 0 \\ 0 & 1 & 0 & 0 \\ 0 & 0 & 1 & 1\end{array}\right], \quad \mathbf{A}_{2}=\mathbf{A}_{1}, \quad \mathbf{A}_{2}^{n}=\mathbf{A}_{1}^{n}$. 
We note that $\mathbf{I}_{33}$ and $\mathbf{I}_{22}$ are the 3-by-3 and 2-by-2 identity matrices, respectively, $\mathbf{0}_{22}$ is the 2-by-2 zero matrix and $\langle\bullet\rangle$ signify the diag function.

On the other hand, by the employment of the Voigt notation for the constitutive laws defined in the second relations of Eqs. (2) and (3) and by considering the plane stress state of the deformation, the classical and double stress vectors are expressed as the following relations:

$$
\begin{aligned}
& \boldsymbol{\sigma}=\left\{\begin{array}{l}
\sigma_{11} \\
\sigma_{22} \\
\tau_{12} \\
\tau_{13} \\
\tau_{23}
\end{array}\right\}=\mathcal{C} \boldsymbol{\epsilon}, \quad \boldsymbol{\Sigma}=\left\{\begin{array}{l}
\boldsymbol{\Sigma}_{1} \\
\boldsymbol{\Sigma}_{2} \\
\boldsymbol{\Sigma}_{3} \\
\boldsymbol{\Sigma}_{4}
\end{array}\right\}=\left[\begin{array}{cccc}
\mathcal{D}_{1} & \mathbf{0} & \mathbf{0} & \mathbf{0} \\
\mathbf{0} & \mathcal{D}_{2} & \mathbf{0} & \mathbf{0} \\
\mathbf{0} & \mathbf{0} & \mathcal{D}_{3} & \mathbf{0} \\
\mathbf{0} & \mathbf{0} & \mathbf{0} & \mathcal{D}_{4}
\end{array}\right]\left\{\begin{array}{l}
\boldsymbol{\eta}_{1} \\
\boldsymbol{\eta}_{2} \\
\boldsymbol{\eta}_{3} \\
\boldsymbol{\eta}_{4}
\end{array}\right\}=\mathcal{D} \boldsymbol{\eta}, \\
& \boldsymbol{\Sigma}_{1}=\left\{\begin{array}{l}
\Sigma_{111} \\
\Sigma_{221} \\
\Sigma_{212}
\end{array}\right\}=\mathcal{D}_{1} \boldsymbol{\eta}_{1}, \quad \boldsymbol{\Sigma}_{2}=\left\{\begin{array}{l}
\Sigma_{222} \\
\Sigma_{112} \\
\Sigma_{121}
\end{array}\right\}=\mathcal{D}_{2} \boldsymbol{\eta}_{2}, \\
& \boldsymbol{\Sigma}_{3}=\left\{\begin{array}{l}
\Sigma_{113} \\
\Sigma_{131} \\
\Sigma_{223} \\
\Sigma_{232}
\end{array}\right\}=\mathcal{D}_{3} \boldsymbol{\eta}_{3}, \quad \boldsymbol{\Sigma}_{4}=\left\{\begin{array}{l}
\Sigma_{123} \\
\Sigma_{231} \\
\Sigma_{312}
\end{array}\right\}=\mathcal{D}_{4} \boldsymbol{\eta}_{4},
\end{aligned}
$$

where $\mathcal{C}$ is the classical material stiffness matrix presented as

$$
\boldsymbol{C}=\left[\begin{array}{ccccc}
\mathcal{C}_{11} & \mathcal{C}_{12} & 0 & 0 & 0 \\
& \mathcal{C}_{22} & 0 & 0 & 0 \\
& & \mathcal{C}_{66} & 0 & 0 \\
& & & \kappa \mathcal{C}_{55} & 0 \\
& & & & \kappa \mathcal{C}_{44}
\end{array}\right]
$$

with $\kappa$ as the shear correction factor for the FSDPT. Besides, $\mathcal{D}_{i}(i=1,2,3,4)$ are the higher-order material stiffness matrices associated with the SG elasticity and can be given as follows:

$$
\begin{aligned}
& \mathcal{D}_{1}=\left[\begin{array}{lll}
d_{11}^{1} & d_{12}^{1} & d_{13}^{1} \\
& d_{22}^{1} & d_{23}^{1} \\
& & d_{33}^{1}
\end{array}\right], \quad \mathcal{D}_{2}=\left[\begin{array}{lll}
d_{11}^{2} & d_{12}^{2} & d_{13}^{2} \\
& d_{22}^{2} & d_{23}^{2} \\
& d_{33}^{2}
\end{array}\right] \\
& \mathcal{D}_{3}=\left[\begin{array}{llll}
d_{11}^{3} & d_{12}^{3} & d_{13}^{3} & d_{14}^{3} \\
& d_{22}^{3} & d_{23}^{3} & d_{24}^{3} \\
& & d_{33}^{1} & d_{34}^{3} \\
& & d_{44}^{3}
\end{array}\right], \quad \mathcal{D}_{4}=\left[\begin{array}{lll}
d_{11}^{4} & d_{12}^{4} & d_{13}^{4} \\
& d_{22}^{1} & d_{23}^{4} \\
& & d_{33}^{4}
\end{array}\right] .
\end{aligned}
$$

The constants $\mathcal{C}_{i j}$ and $d_{i j}^{k}$ in Eqs. (21) and (22) can be determined by using generalized numerical homogenization procedures for any specific microarchitecture lattices. 


\section{Hamilton's Principle}

Hamilton's principle is applied to present the dynamic governing equations. By setting $\delta \Pi, \delta \mathcal{T}$, and $\delta \mathcal{W}$, respectively, for strain energy, kinetic energy, and the work done by external loads, where $\delta$ stands for the first variation operator, it can be expressed in the form

$\int_{t_{1}}^{t_{2}}(\delta \mathcal{T}-\delta \Pi+\delta \mathcal{W}) d t=0$.

Under the strain energy of Eq. (1) and by following constitutive relations of Eq. (20), the total strain energy related to the SG theory are represented as

$$
\begin{aligned}
\delta \Pi & =\int_{\mathcal{V}}\left(\delta \boldsymbol{\epsilon}^{\mathrm{T}} \boldsymbol{\sigma}+\delta \boldsymbol{\eta}^{\mathrm{T}} \boldsymbol{\Sigma}\right) d \mathcal{V} \\
& =\int_{\mathcal{V}}\left(\delta \boldsymbol{\epsilon}^{\mathrm{T}} \boldsymbol{C} \boldsymbol{\epsilon}+\delta \boldsymbol{\eta}_{1}^{\mathrm{T}} \mathcal{D}_{1} \boldsymbol{\eta}_{1}+\delta \boldsymbol{\eta}_{2}^{\mathrm{T}} \mathcal{D}_{2} \boldsymbol{\eta}_{2}+\delta \boldsymbol{\eta}_{3}^{\mathrm{T}} \mathcal{D}_{3} \boldsymbol{\eta}_{3}+\delta \boldsymbol{\eta}_{4}^{\mathrm{T}} \mathcal{D}_{4} \boldsymbol{\eta}_{4}\right) d \mathcal{V} .
\end{aligned}
$$

It is worth noting that by considering the geometrically nonlinear kinematic relations of Eq. (1), the first variations of the strain and SG vectors are defined as follows:

$\delta \boldsymbol{\epsilon}=\left(\mathbf{A E}+\mathbf{A}^{n} \mathbf{E}^{n}\right) \delta \mathbf{u}$,

$\delta \boldsymbol{\eta}_{1}=\left(\mathbf{A}_{1} \mathbf{E}_{1}+\mathbf{A}_{1}^{n} \mathbf{E}_{1}^{n}\right) \delta \mathbf{u}, \quad \delta \boldsymbol{\eta}_{2}=\left(\mathbf{A}_{2} \mathbf{E}_{2}+\mathbf{A}_{2}^{n} \mathbf{E}_{2}^{n}\right) \delta \mathbf{u}$,

$\delta \mathbf{\kappa}_{3}=\mathbf{E}_{3} \delta \mathbf{u}, \quad \delta \mathbf{\eta}_{4}=\mathbf{E}_{4} \delta \mathbf{u}$.

Besides, by using Eq. (4) and by taking the matrix form of the displacement field proposed in Eq. (6) into account, along with the assumption of $d_{i j}=\gamma \delta_{i j}$ where $\gamma$ denotes the intrinsic inertial length scale, the first variation of the kinetic energy reads as

$\delta \mathcal{T}=\int_{\mathcal{V}}\left(\delta \dot{\mathbf{u}}^{\mathrm{T}} \mathbf{A}_{0}^{\mathrm{T}} \rho \mathbf{A}_{0} \dot{\mathbf{u}}+\gamma \delta \dot{\mathbf{u}}^{\mathrm{T}} \mathbf{L}^{\mathrm{T}} \mathbf{A}_{3}^{\mathrm{T}} \rho \mathbf{A}_{3} \mathbf{L} \dot{\mathbf{u}}\right) d x_{3} d A=\int_{A}\left(\delta \dot{\mathbf{u}}^{\mathrm{T}} \mathbf{m}_{0} \dot{\mathbf{u}}+\gamma \delta \dot{\mathbf{u}}^{\mathrm{T}} \mathbf{L}^{\mathrm{T}} \mathbf{m}_{1} \mathbf{L} \dot{\mathbf{u}}\right) d A$

in which

$\begin{aligned} \mathbf{L}^{\mathrm{T}} & =\left[\begin{array}{cccccccccccc}\partial_{1} & 0 & 0 & \partial_{2} & 0 & 0 & 0 & 0 & 0 & 0 & 0 & 0 \\ 0 & \partial_{2} & \partial_{1} & 0 & 0 & 0 & 0 & 0 & 0 & 0 & 0 & 0 \\ 0 & 0 & 0 & 0 & \partial_{1} & \partial_{2} & 0 & 0 & 0 & 0 & 0 & 0 \\ 0 & 0 & 0 & 0 & 0 & 0 & 1 & 0 & \partial_{1} & 0 & 0 & \partial_{2} \\ 0 & 0 & 0 & 0 & 0 & 0 & 0 & 1 & 0 & \partial_{2} & \partial_{1} & 0\end{array}\right] \\ \mathbf{m}_{0} & =\varrho \int_{-h / 2}^{h / 2} \mathbf{A}_{0}^{\mathrm{T}} \rho \mathbf{A}_{0} d x_{3}, \\ \mathbf{m}_{1} & =\varrho \int_{-h / 2}^{h / 2} \mathbf{A}_{3}^{\mathrm{T}} \rho \mathbf{A}_{3} d x_{3}\end{aligned}$

with

$\mathbf{A}_{3}=\left[\begin{array}{ccc}\mathbf{I}_{44} & \mathbf{0} & x_{3} \mathbf{I}_{44} \\ \mathbf{0} & \mathbf{I}_{44} & \mathbf{0}\end{array}\right]$

where $\varrho$ captures the geometrical density of the lattice architecture.

On the other hand, the variation of the work of the external loads is given by the relation 
$\delta \mathcal{W}=\int_{A} \delta \mathbf{u}^{\mathrm{T}} \mathbf{q} d A$

considering only the transverse loads $\mathbf{q}=\left[\begin{array}{lllll}0 & 0 & f & 0 & 0\end{array}\right]$ indicating the vector of surface forces. The next section provides detailed formulations on the FE discretization procedure for the nonlinear vibration problem of the SG plate model.

\section{Finite element discretization procedure}

In accordance with the basic idea of the FE analysis, the displacement field can be approximated in each element by using appropriate approximate functions:

$\mathbf{u}=\left[\begin{array}{l}u_{1} \\ u_{2} \\ u_{3} \\ \psi_{1} \\ \psi_{2}\end{array}\right]=\mathbb{N} u$

where $u$ is the vector of the nodal unknowns related to the displacement components and

$\mathbb{N}=\left[\begin{array}{llll}\mathbf{N}_{1} & \mathbf{N}_{2} & \ldots & \mathbf{N}_{m}\end{array}\right], \quad \mathbf{N}_{i}=\left[\begin{array}{ccccc}\mathcal{N}_{i} & 0 & 0 & 0 & 0 \\ 0 & \mathcal{N}_{i} & 0 & 0 & 0 \\ 0 & 0 & \mathcal{N}_{i} & 0 & 0 \\ 0 & 0 & 0 & \mathcal{N}_{i} & 0 \\ 0 & 0 & 0 & 0 & \mathcal{N}_{i}\end{array}\right]$

where $\mathcal{N}_{i}$ are the approximate functions and $m$ signifies the number of degrees of freedom (DOF) per node. By using the approximate expression presented for the displacement vector and by substituting Eq. (30) into (12), the approximations of the strain and strain gradient vectors within each element are expressed by

$\boldsymbol{\epsilon}=\left(\mathbf{A B}+\frac{1}{2} \mathbf{A}^{n} \mathcal{B}^{n}\right)$ un,

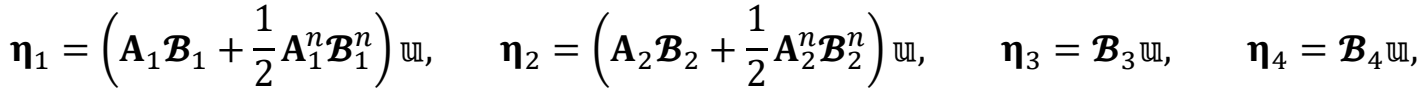

where the linear $(\boldsymbol{B})$ and nonlinear $\left(\mathcal{B}^{n}\right)$ FE matrix operators of the classical theory are given as

$\mathcal{B}=\mathbf{E N}, \quad \mathcal{B}^{n}=\left\langle\boldsymbol{Q}_{\text {}}\right\rangle \overline{\boldsymbol{Q}}+\left\langle\overline{\boldsymbol{Q}}_{\text {}}\right\rangle \boldsymbol{Q}$,

$\boldsymbol{Q}=\mathbf{Q} \mathbb{N}, \quad \overline{\mathcal{Q}}=\overline{\mathbf{Q}} \mathbb{N}$,

while for the SG counterparts, they can be written as

$\mathcal{B}_{1}=\mathbf{E}_{1} \mathbb{N}, \quad \mathcal{B}_{1}^{n}=\left\langle\boldsymbol{Q}_{1}\right.$ 凹 $\rangle \overline{\boldsymbol{Q}}_{1}+\left\langle\overline{\boldsymbol{Q}}_{1}\right.$ 匹 $\rangle \boldsymbol{Q}_{1}$,

$\mathcal{B}_{2}=\mathbf{E}_{2} \mathbb{N}, \quad \boldsymbol{B}_{2}^{n}=\left\langle\boldsymbol{Q}_{2}\right.$ 凹ा $\rangle \overline{\boldsymbol{Q}}_{2}+\left\langle\overline{\boldsymbol{Q}}_{2}\right.$ 凹 $\rangle \boldsymbol{Q}_{2}$,

$\mathcal{B}_{3}=\mathbf{E}_{3} \mathbb{N}, \quad \mathcal{B}_{4}=\mathbf{E}_{4} \mathbb{N}$,

$\boldsymbol{Q}_{i}=\mathbf{Q}_{i} \mathbb{N}, \quad \overline{\boldsymbol{Q}}_{i}=\overline{\mathbf{Q}}_{i} \mathbb{N} \quad(i=1,2)$. 
By following Eq. (25), the corresponding first variation of the relations in Eq. (32) can be given by the relations

$\delta \boldsymbol{\epsilon}=\left(\mathbf{A B}+\mathbf{A}^{n} \mathcal{B}^{n}\right) \delta$ un,

$\delta \boldsymbol{\eta}_{1}=\left(\mathbf{A}_{1} \mathcal{B}_{1}+\mathbf{A}_{1}^{n} \mathcal{B}_{1}^{n}\right) \delta$ un, $\quad \delta \boldsymbol{\eta}_{2}=\left(\mathbf{A}_{2} \mathcal{B}_{2}+\mathbf{A}_{2}^{n} \mathcal{B}_{2}^{n}\right) \delta$ un, $\quad \delta \boldsymbol{\eta}_{3}=\mathcal{B}_{3} \delta$ un, $\quad \delta \boldsymbol{\eta}_{4}=\mathcal{B}_{4} \delta$ uा.

Now, by substituting Eqs. (32) and (35) into the variation of the strain energy functional Eq. (24), FE approximations of the energy is presented as

$$
\begin{aligned}
& \delta \Pi=\int_{A} \delta \text { uा }^{\mathrm{T}}\left(\mathcal{B}^{\mathrm{T}} \overline{\mathcal{C}}_{1} \mathcal{B}+\frac{1}{2} \mathcal{B}^{\mathrm{T}} \overline{\mathcal{C}}_{2} \mathcal{B}^{n}+\mathcal{B}^{n \mathrm{~T}} \overline{\mathcal{C}}_{2}^{\mathrm{T}} \mathcal{B}+\frac{1}{2} \mathcal{B}^{n \mathrm{~T}} \overline{\mathcal{C}}_{3} \mathcal{B}^{n}\right) \text { uा } d A \\
& +\int_{A} \delta \mathrm{un}^{\mathrm{T}}\left(\mathcal{B}_{1}^{\mathrm{T}} \overline{\mathcal{D}}_{1} \mathcal{B}_{1}+\mathcal{B}_{2}^{\mathrm{T}} \overline{\mathcal{D}}_{2} \mathcal{B}_{2}+\mathcal{B}_{3}^{\mathrm{T}} \overline{\mathcal{D}}_{3} \mathcal{B}_{3}+\mathcal{B}_{4}^{\mathrm{T}} \overline{\mathcal{D}}_{4} \mathcal{B}_{4}+\frac{1}{2} \mathcal{B}_{1}^{\mathrm{T}} \overline{\mathcal{D}}_{5} \mathcal{B}_{1}^{n}+\frac{1}{2} \mathcal{B}_{2}^{\mathrm{T}} \overline{\mathcal{D}}_{6} \mathcal{B}_{2}^{n}\right. \\
& \left.+\mathcal{B}_{1}^{n \mathrm{~T}} \overline{\mathcal{D}}_{5}^{\mathrm{T}} \mathcal{B}_{1}+\mathcal{B}_{2}^{n \mathrm{~T}} \overline{\mathcal{D}}_{6}^{\mathrm{T}} \mathcal{B}_{2}+\frac{1}{2} \mathcal{B}_{1}^{n \mathrm{~T}} \overline{\mathcal{D}}_{7} \mathcal{B}_{1}^{n}+\frac{1}{2} \mathcal{B}_{2}^{n \mathrm{~T}} \overline{\mathcal{D}}_{8} \mathcal{B}_{2}^{n}\right) \text { u } d A
\end{aligned}
$$

where the first and second integrals capture the classical and SG effects, respectively. The following material matrices, produced by integrations over the thickness direction, are written as

$$
\begin{array}{ll}
\overline{\mathcal{C}}_{1}= & \int_{-h / 2}^{h / 2} \mathbf{A}^{\mathrm{T}} \boldsymbol{C} \mathbf{A} d x_{3}, \quad \overline{\mathcal{C}}_{2}=\int_{-h / 2}^{h / 2} \mathbf{A}^{\mathrm{T}} \boldsymbol{C} \mathbf{A}^{n} d x_{3}, \quad \overline{\mathcal{C}}_{3}=\int_{-h / 2}^{h / 2} \mathbf{A}^{n \mathrm{~T}} \boldsymbol{C} \mathbf{A}^{n} d x_{3} \\
\overline{\mathcal{D}}_{1}= & \int_{-h / 2}^{h / 2} \mathbf{A}_{1}^{\mathrm{T}} \mathcal{D}_{1} \mathbf{A}_{1} d x_{3}, \quad \overline{\mathcal{D}}_{2}=\int_{-h / 2}^{h / 2} \mathbf{A}_{2}^{\mathrm{T}} \mathcal{D}_{2} \mathbf{A}_{2} d x_{3}, \quad \overline{\mathcal{D}}_{3}=\int_{-h / 2}^{h / 2} \mathcal{D}_{3} d x_{3}, \\
\overline{\mathcal{D}}_{4}= & \int_{-h / 2}^{h / 2} \mathcal{D}_{4} d x_{3}, \quad \overline{\mathcal{D}}_{5}=\int_{-h / 2}^{h / 2} \mathbf{A}_{1}^{\mathrm{T}} \mathcal{D}_{1} \mathbf{A}_{1}^{n} d x_{3}, \quad \overline{\mathcal{D}}_{6}=\int_{-h / 2}^{h / 2} \mathbf{A}_{2}^{\mathrm{T}} \mathcal{D}_{2} \mathbf{A}_{2}^{n} d x_{3} \\
\overline{\mathcal{D}}_{7}= & \int_{-h / 2}^{h / 2} \mathbf{A}_{1}^{n \mathrm{~T}} \mathcal{D}_{1} \mathbf{A}_{1}^{n} d x_{3}, \overline{\mathcal{D}}_{8}=\int_{-h / 2}^{h / 2} \mathbf{A}_{2}^{n \mathrm{~T}} \mathcal{D}_{2} \mathbf{A}_{2}^{n} d x_{3} .
\end{array}
$$

Besides, substituting Eq. (30) into (26) and (29) leads to the relations for FE approximations of the variations of the kinetic energy and work of external loads:

$$
\begin{aligned}
& \delta \mathcal{T}=\int_{A} \delta \dot{u n}^{\mathrm{T}}\left(\mathbb{N}^{\mathrm{T}} \mathbf{m}_{0} \mathbb{N}+\gamma \mathcal{L}^{\mathrm{T}} \mathbf{m}_{1} \mathcal{L}\right) \dot{\mathrm{u}} d A \\
& \delta \mathcal{W}=\int_{A} \delta \text { un }^{\mathrm{T}} \mathbb{N}^{\mathrm{T}} \mathbf{q} d A
\end{aligned}
$$

where $\mathcal{L}=\mathbf{L N}$.

Finally, substituting the fundamental equations given in Eqs. (36) and (38) into Hamilton's principle, results in the nonlinear FE governing equation:

$\mathbf{M u ̈ i}+\mathbf{K} u+F=\mathbf{0}$ 
in which the stiffness matrix $\mathbf{K}=\mathbf{K}_{l}+\mathbf{K}_{n l}$ addresses both linear $\left(\mathbf{K}_{l}\right)$ and nonlinear parts $\left(\mathbf{K}_{n l}\right)$ where the linear stiffness matrix consists of two terms, i.e., $\mathbf{K}_{l}=\mathbf{K}_{l}^{c}+\mathbf{K}_{l}^{s}$ with

$\mathbf{K}_{l}^{c}=\int_{A}\left(\mathcal{B}^{\mathrm{T}} \overline{\boldsymbol{C}}_{1} \mathcal{B}+\frac{1}{2} \mathcal{B}^{\mathrm{T}} \overline{\boldsymbol{C}}_{2} \mathcal{B}^{n}+\mathcal{B}^{n \mathrm{~T}} \overline{\mathcal{C}}_{2}^{\mathrm{T}} \mathcal{B}+\frac{1}{2} \mathcal{B}^{n \mathrm{~T}} \overline{\boldsymbol{C}}_{3} \mathcal{B}^{n}\right) d A$

$\mathbf{K}_{l}^{s}=\int_{A}\left(\mathcal{B}_{1}^{\mathrm{T}} \overline{\mathcal{D}}_{1} \mathcal{B}_{1}+\mathcal{B}_{2}^{\mathrm{T}} \overline{\mathcal{D}}_{2} \mathcal{B}_{2}+\mathcal{B}_{3}^{\mathrm{T}} \overline{\mathcal{D}}_{3} \mathcal{B}_{3}+\mathcal{B}_{4}^{\mathrm{T}} \overline{\mathcal{D}}_{4} \mathcal{B}_{4}\right) d A$

where superscripts $c$ and $s$, respectively, signify the classical and SG parts. In a similar manner, the nonlinear stiffness matrix can be divided into two parts, i.e., $\mathbf{K}_{n l}=\mathbf{K}_{n l}^{c}+\mathbf{K}_{n l}^{s}$ with

$\mathbf{K}_{n l}^{c}=\frac{1}{2} \int_{A}\left(\mathcal{B}^{\mathrm{T}} \overline{\mathcal{C}}_{2} \mathcal{B}^{n}+2 \mathcal{B}^{n \mathrm{~T}} \overline{\mathcal{C}}_{2}^{\mathrm{T}} \mathcal{B}+\mathcal{B}^{n \mathrm{~T}} \overline{\boldsymbol{C}}_{3} \mathcal{B}^{n}\right) d A$

$\mathbf{K}_{n l}^{s}=\frac{1}{2} \int_{A}\left(\mathcal{B}_{1}^{\mathrm{T}} \overline{\mathcal{D}}_{5} \mathcal{B}_{1}^{n}+\mathcal{B}_{2}^{\mathrm{T}} \overline{\mathcal{D}}_{6} \mathcal{B}_{2}^{n}+2 \mathcal{B}_{1}^{n \mathrm{~T}} \overline{\mathcal{D}}_{5}^{\mathrm{T}} \mathcal{B}_{1}+2 \mathcal{B}_{2}^{n \mathrm{~T}} \overline{\mathcal{D}}_{6}^{\mathrm{T}} \mathcal{B}_{2}+\mathcal{B}_{1}^{n \mathrm{~T}} \overline{\mathcal{D}}_{7} \mathcal{B}_{1}^{n}+\mathcal{B}_{2}^{n \mathrm{~T}} \overline{\mathcal{D}}_{8} \mathcal{B}_{2}^{n}\right) d A$

In addition, the mass matrix and force vector are defined as

$\mathbf{M}=\int_{A}\left(\mathbb{N}^{\mathrm{T}} \mathbf{m}_{0} \mathbb{N}+\gamma \mathcal{L}^{\mathrm{T}} \mathbf{m}_{1} \mathcal{L}\right) d A$,

$\mathbf{F}=\int_{A} \mathbb{N}^{\mathrm{T}} \mathbf{f} d A$

By considering the harmonic excitation under a spatially uniform transverse load with an excitation frequency $\omega$, and taking dissipation into account through the external viscous damping effect, the FE governing equation can be rewritten as follows:

$\mathbf{M} \ddot{u} \mathbf{l}+\mathbf{C} \ddot{u}+\mathbf{K} \ddot{u}+\mathbf{F} \cos (\omega t)=\mathbf{0}$

with $\mathbf{C}$ as the damping matrix defined as

$\mathbf{C}=c \mathbf{K}_{l} / \omega_{L}$

where $c$ is the damping coefficient and $\omega_{L}$ is the linear fundamental frequency of the structure. The nonlinear FE governing equation provided in Eq. (44) can be solved by the numerical solution procedure described in [56,57] in which the time-periodic differential operators and an arc-length continuation technique are employed to present the nonlinear free/forced vibration responses. The stiffness and mass matrices as well as the force vector defined in Eqs. (40)-(43) are obtained by employing a quasiconforming six-node triangular element detailed next.

\section{Quasi-conforming higher-order triangular element}

In the majority of engineering applications, the standard $C^{0}$-continuous $2 \mathrm{D}$ and $3 \mathrm{D}$ elements can be effectively employed and, accordingly, different commercial FE software can be utilized for numerical simulations. However, SG elasticity requires, due to the existence of the higher-order derivations of the field variables in the energy functional, either $C^{1}$-continuous elements or $C^{0}$-continuous elements but a 
mixed formulation. The present study follows the former route by adopting a higher-order element: a quasi-conforming six-node triangular element.

To present the fundamentals of the element, the local area (LA) coordinate system of $L_{1}, L_{2}, L_{3}$ is employed. Besides the value of the field variables, their first-order derivatives are considered as additional nodal values. To give more details about the element, let us consider $\varphi$ as a scalar field. As explained above, the values of scalar field $\left(\Phi_{i}^{0}\right)$ and its first-order derivatives with respect to $x_{1}$ and $x_{2}$ $\left(\Phi_{i}^{1}, \Phi_{i}^{2}\right)$ are taken as the nodal values, as demonstrated in Figure 1.

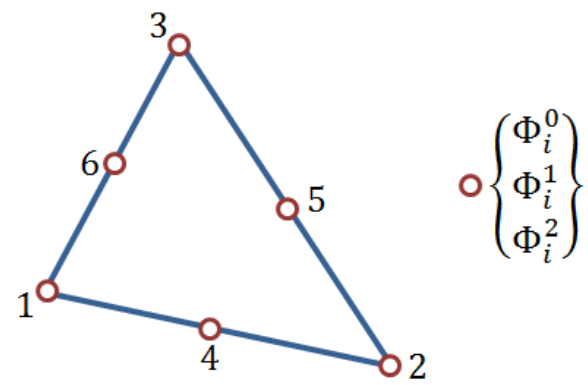

Figure 1: Description of the quasi-conforming 6-node triangular element

We note that $i$ stands for the number of nodes, and since a six-node triangle is considered, $i=1,2, \ldots, 6$. The associated mathematical formulations for the described nodal values are

$\varphi\left(\mathbf{L}_{i}\right)=\Phi_{i}, \quad \frac{\partial \varphi\left(\mathbf{L}_{i}\right)}{\partial x_{1}}=\Phi_{i}^{1}, \quad \frac{\partial \varphi\left(\mathbf{L}_{i}\right)}{\partial y}=\Phi_{i}^{2}, \quad(i=1,2, \ldots, 6)$

with $\mathbf{L}_{i}=\left[\begin{array}{lll}L_{1}^{i} & L_{2}^{i} & L_{3}^{i}\end{array}\right]$ as the vector of the LA coordinates at node $i$. It is now worth noting that by considering three nodal values for each field variable $\left(\Phi^{0}, \Phi^{1}, \Phi^{2}\right)$ and having six nodes in the triangular element, there are 18 DOFs for each variable. By following the proposed nodal values, the sample field variable $\varphi$ can be approximated by an 18-term polynomial in terms of $L_{1}, L_{2}, L_{3}$ as

$$
\begin{aligned}
\varphi & =\alpha_{1} L_{1}+\alpha_{2} L_{2}+\alpha_{3} L_{3}+\alpha_{4} L_{1} L_{2}+\alpha_{5} L_{2} L_{3}+\alpha_{6} L_{1} L_{3}+\alpha_{7} L_{1}^{2} L_{2}+\alpha_{8} L_{2}^{2} L_{3}+\alpha_{9} L_{3}^{2} L_{1} \\
& +\alpha_{10} L_{1}^{2} L_{2}^{2}+\alpha_{11} L_{2}^{2} L_{3}^{2}+\alpha_{12} L_{3}^{2} L_{1}^{2}+\alpha_{13} L_{1}^{3} L_{2}+\alpha_{14} L_{2}^{3} L_{3}+\alpha_{15} L_{3}^{3} L_{1}+\alpha_{16} L_{1}^{4} L_{2}+\alpha_{17} L_{2}^{4} L_{3} \\
& +\alpha_{18} L_{3}^{4} L_{1}
\end{aligned}
$$

which can be represented in the vector form

$\varphi=\boldsymbol{\Theta}(\mathbf{L}) \boldsymbol{\alpha}$.

with the row vector of

$$
\begin{aligned}
& \boldsymbol{\Theta}(\mathbf{L})=\left[\begin{array}{lllllllllllllllllllll}
L_{1} & L_{2} & L_{3} & L_{1} L_{2} & L_{2} L_{3} & L_{1} L_{3} & L_{1}^{2} L_{2} & L_{2}^{2} L_{3} & L_{3}^{2} L_{1} & L_{1}^{2} L_{2}^{2} & L_{2}^{2} L_{3}^{2} & L_{3}^{2} L_{1}^{2}
\end{array}\right. \\
& \left.\begin{array}{llllll}
L_{1}^{3} L_{2} & L_{2}^{3} L_{3} & L_{3}^{3} L_{1} & L_{1}^{4} L_{2} & L_{2}^{4} L_{3} & L_{3}^{4} L_{1}
\end{array}\right],
\end{aligned}
$$

and the column vector of

$\boldsymbol{\alpha}=\left[\begin{array}{llll}\alpha_{1} & \alpha_{2} & \ldots & \alpha_{18}\end{array}\right]^{\mathrm{T}}$. 
Since the problem was formulated within the FSDPT with five field variables, i.e., three displacements $\left(u_{1}, u_{2}, u_{3}\right)$ and two rotations $\left(\psi_{1}, \psi_{2}\right)$, the proposed element has a total of 90 DOFs. Accordingly, the approximation of Eq. (47) or (48) can be substituted into nodal values conditions of Eq. (46) which leads to the following relation

$\Phi=\mathbf{Y} \boldsymbol{\alpha}$,

with

$\boldsymbol{\Phi}=\left[\begin{array}{llllllllll}\Phi_{1}^{0} & \Phi_{1}^{1} & \Phi_{1}^{2} & \Phi_{2}^{0} & \Phi_{2}^{1} & \Phi_{2}^{2} & \ldots & \Phi_{6}^{0} & \Phi_{6}^{1} & \Phi_{6}^{2}\end{array}\right]^{\mathrm{T}}$,

$\mathbf{Y}=\left[\begin{array}{c}\boldsymbol{\Lambda}\left(\mathbf{L}_{1}\right) \\ \boldsymbol{\Lambda}\left(\mathbf{L}_{2}\right) \\ \vdots \\ \boldsymbol{\Lambda}\left(\mathbf{L}_{6}\right)\end{array}\right]_{18 \times 18}, \quad \boldsymbol{\Lambda}\left(\mathbf{L}_{i}\right)=\left[\begin{array}{c}\mathbf{\Theta}\left(\mathbf{L}_{i}\right) \\ \frac{\partial \boldsymbol{\Theta}\left(\mathbf{L}_{\boldsymbol{i}}\right)}{\partial x_{1}} \\ \frac{\partial \boldsymbol{\Theta}\left(\mathbf{L}_{\boldsymbol{i}}\right)}{\partial x_{2}}\end{array}\right]_{3 \times 18}$

By using Eq. (51), the column vector $\boldsymbol{\alpha}$ can be determined as $\boldsymbol{\alpha}=\mathbf{Y}^{\mathbf{- 1}} \boldsymbol{\Phi}$, and then by following Eq. (48), the approximation of the sample field variable is obtained as follows:

$\phi=\boldsymbol{\Theta}(\mathbf{L}) \mathbf{Y}^{-1} \boldsymbol{\Phi}=\mathbf{N} \boldsymbol{\Phi}$,

where $\mathbf{N}=\left[\begin{array}{llll}\mathcal{N}_{1} & \mathcal{N}_{2} & \ldots & \mathcal{N}_{18}\end{array}\right]=\boldsymbol{\Theta}(\mathbf{L}) \mathbf{Y}^{-1}$ introduces the vector of approximate functions which can be used in Eq. (31) for the approximation functions. The Gauss quadrature can be applied to finally calculate the stiffness and mass matrices along with the force vector given in Eqs. (40)-(43).

\section{Numerical results}

The nonlinear shear deformation SG plate model, discretized by the quasi-conforming higher-order triangular finite element, will be next applied for studying the microarchitecture-dependent free and forced vibration of cellular plate structures. By employing the SG plate model, the mechanical behavior of three-dimensional cellular structures with prismatic lattice microarchitectures can be modeled by a two-dimensional model significantly reducing the computational cost of the numerical structural analysis.

An example of the cellular plates with an equitriangular lattice architecture is shown in Figure 2 and geometrical parameters are given in Table 1. The SG plate model with the constitutive parameters (as presented in the material matrices $\mathcal{C}, \mathcal{D}_{1}, \mathcal{D}_{2}, \mathcal{D}_{3}, \mathcal{D}_{4}$ in Eqs. (20)-(22) and further in (24)) can be determined by using a homogenization technique in which the deformations of the 2D SG plate model are compared to the reference full-field model of the classical three-dimensional continuum mechanics. For the specific equitriangular lattice architecture considered in the present work, Kakalo and Niiranen [48] have determined the classical and SG material parameters for a reduced SG plate theory, augmented by Torabi and Niiranen [49]. The nonzero constitutive parameters are given in Tables 2 and 3. Since the cellular plate is assumed to be made of stainless steel, the mass density is $\rho=7800\left(\mathrm{~kg} / \mathrm{m}^{3}\right)$, and by 
considering the geometrical parameters of the unit cell (see Figure 2b), the value of the factor used in Eq. (27) is $\varrho=0.3$.

(a)



(b)

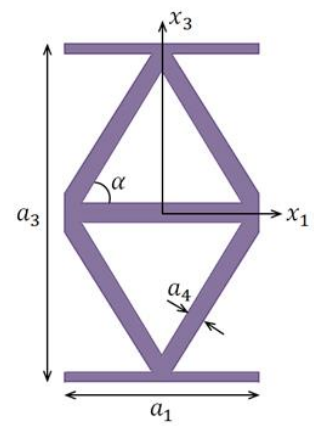

Figure 2: (a) The schematics of a cellular plate with a triangularly prismatic core and (b) the related plane geometry of a unit cell

Table 1: The geometries of the unit cell demonstrated in Figure $2 b$

\begin{tabular}{cccc}
\hline$a_{1}(\mathrm{~mm})$ & $a_{3}(\mathrm{~mm})$ & $a_{4}(\mathrm{~mm})$ & $\alpha^{\circ}$ \\
\hline 5 & 8.66 & 0.5 & 60 \\
\hline
\end{tabular}

Table 2: Classical overall constitutive parameters (GPa) for the proposed equitriangular lattice architecture [48]

\begin{tabular}{cccccc}
\hline $\mathcal{C}_{11}$ & $\mathcal{C}_{12}$ & $\mathcal{C}_{22}$ & $\mathcal{C}_{44}$ & $\mathcal{C}_{55}$ & $\mathcal{C}_{66}$ \\
\hline 26.27 & 6.57 & 64.96 & 14.43 & 9.76 & 14.43 \\
\hline
\end{tabular}

Table 3: Non-classical SG constitutive parameters $(\mathrm{kN})[48,49]$

\begin{tabular}{cc}
\hline$d_{11}^{1}=d_{22}^{2}=d_{11}^{3}$ & 63.96 \\
\hline$d_{12}^{1}=d_{12}^{2}=d_{13}^{3}$ & 17.59 \\
\hline$d_{22}^{1}=d_{11}^{2}=d_{33}^{3}$ & 50.30 \\
\hline$d_{33}^{1}=d_{33}^{2}=d_{11}^{4}$ & 17.78 \\
\hline$d_{22}^{3}=d_{33}^{4}$ & 12.02 \\
\hline$d_{44}^{3}=d_{22}^{4}$ & 17.78 \\
\hline
\end{tabular}

To show the applicability of the proposed FE element and solution procedure, the results of the largeamplitude free/forced vibration are represented for diverse shapes of plate mid-surfaces, i.e., a rectangle, circle, and an annular sector. The schematics of the plate mid-surfaces and the associated geometries are given in Figure 3. Since the main objective of the study is to analyze the microarchitecture effects of the cellular plates with different numbers of lattice layers on the vibration behavior, rectangular plates with one, two, and three lattice layers are presented in Figure 4 to highlight the idea of the layering starting from one layer $(N=1)$. 

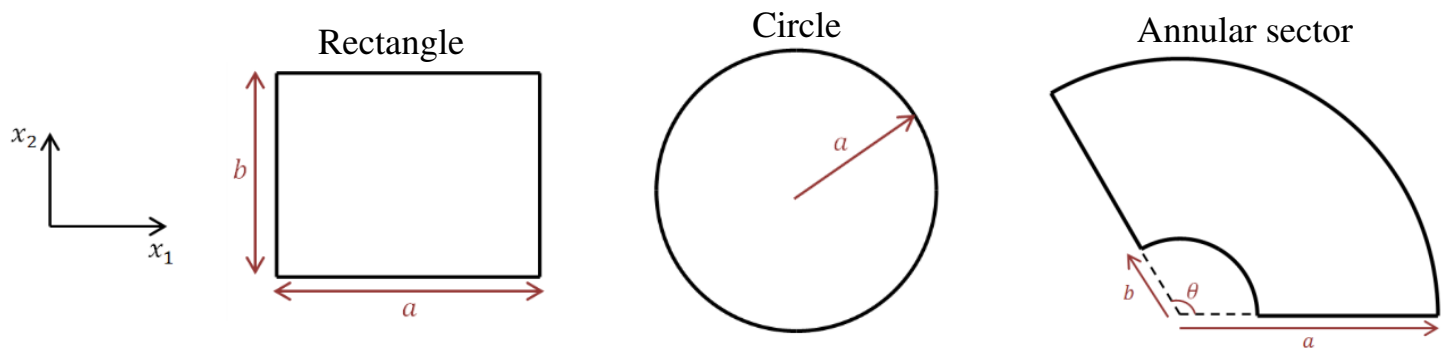

Figure 3: Schematics and geometrical parameters of the mid-surfaces for different plate geometries
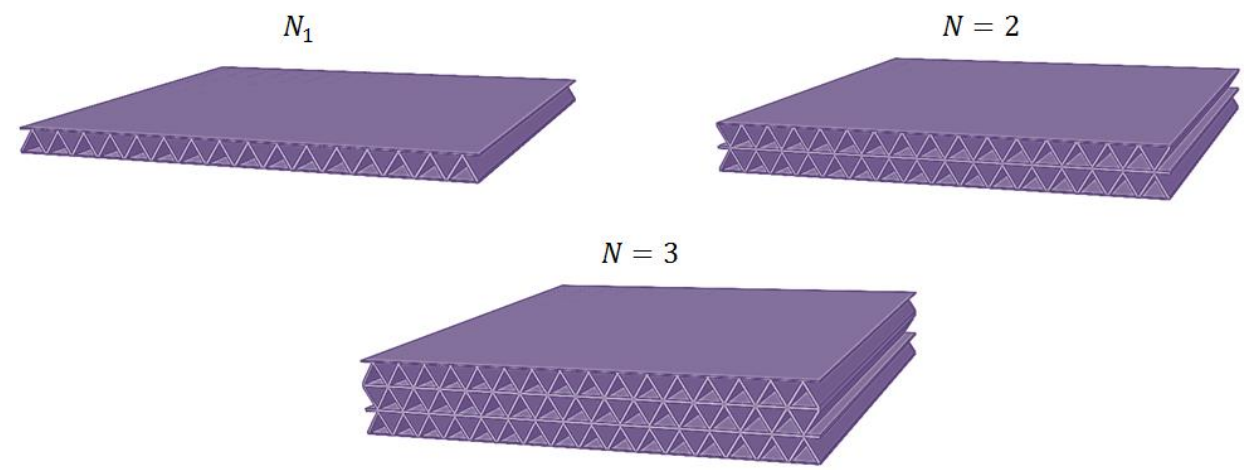

Figure 4: Plates with different numbers $(N)$ of lattice layers

For the numerical examples, clamped (C) and simply supported (S) constraints are:

clamped:

simply supported:

$$
\begin{aligned}
& u_{n}=u_{t}=u_{3}=\Psi_{n}=\Psi_{t}=0, \\
& u_{n}=u_{t}=u_{3}=\Psi_{t}=0
\end{aligned}
$$

where the normal and tangential components of the displacements and rotation are given by the relations $u_{n}=m_{1} u_{1}+m_{2} u_{2}, u_{t}=-m_{2} u_{1}+m_{1} u_{2}, \Psi_{n}=m_{1} \psi_{1}+m_{2} \psi_{2}$ and $\Psi_{t}=-m_{2} \psi_{1}+m_{1} \psi_{2}$ with $m_{1}$ and $m_{2}$ as the unit normal vector components.

Before going through the details of the numerical results, the impacts of the micro inertia are studied to see how significantly this term affects the dynamic analysis of lattice structures. For this purpose, the variations of the linear frequency parameters $\left(\Omega=\omega_{L} a^{2} / h \sqrt{\varrho \rho / \mathcal{C}_{11}}\right)$ of the square lattice plate versus the mode numbers are presented in Figure 5 for different values of the intrinsic inertial length scale parameter $\gamma$ (see Eqs. (4), (26) and (38)). The single-layer lattice plate with fully clamped boundary conditions is taken as an example. To give more quantitative results, the values of the first five frequency values are tabulated in Table 4. It is seen that the inertial length scale corresponding to the micro inertia term does not considerably affect the lower modes of the frequency parameter (at least for mode numbers lower than 10). Based on this finding, the micro inertia term is neglected in what follows, i.e., $\gamma=0$. 


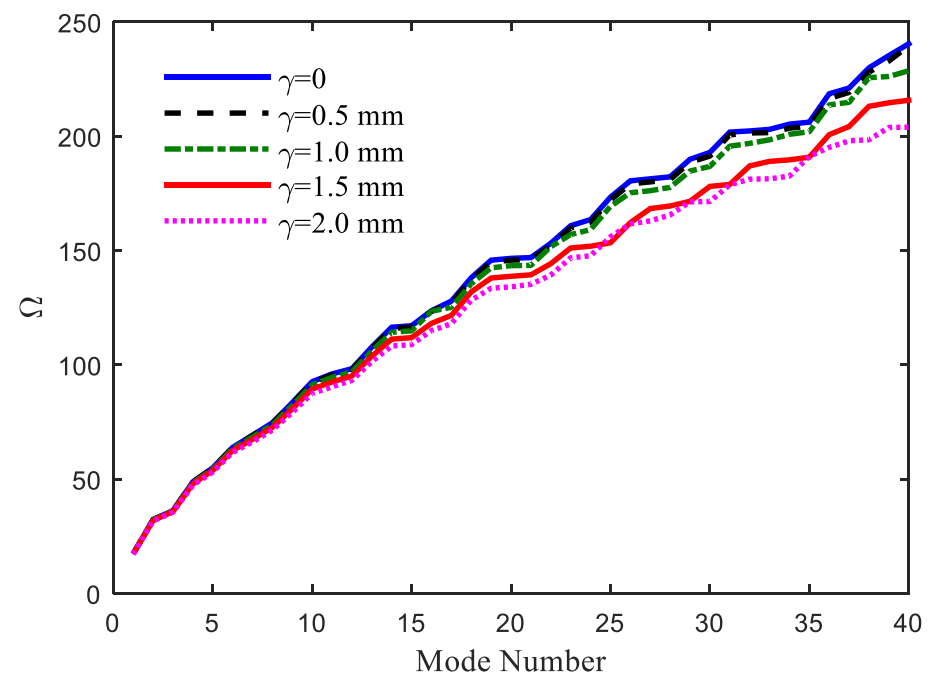

Figure 5: The impact of the inertial length scale on linear frequency values of a one-layer cellular square plate with clamped boundaries $(a / h=23.1)$

Table 4: The effect of the inertial length scale on the first five linear frequency values of a one-layer cellular square plate with clamped boundaries $(a / h=23.1)$

\begin{tabular}{cccccc}
\hline & \multicolumn{5}{c}{$\gamma(\mathrm{mm})$} \\
\cline { 2 - 6 } & 0 & 0.5 & 1 & 1.5 & 2 \\
\hline$\Omega_{1}$ & 17.296 & 17.287 & 17.258 & 17.211 & 17.145 \\
$\Omega_{2}$ & 32.263 & 32.222 & 32.103 & 31.906 & 31.636 \\
$\Omega_{3}$ & 36.188 & 36.143 & 36.008 & 35.787 & 35.484 \\
$\Omega_{4}$ & 48.750 & 48.658 & 48.386 & 47.941 & 47.336 \\
$\Omega_{5}$ & 54.894 & 54.772 & 54.408 & 53.816 & 53.017 \\
\hline
\end{tabular}

\subsection{Convergence analysis and model comparisons}

This section provides some numerical results to express the accuracy and efficiency of the SG plate model and the employed FE method. First of all, the convergence rates of the relative errors of the linear fundamental frequency for the one- and two-layer plates versus an increase of the number of the elements are demonstrated in Figures 6 (clamped square, CCCC; simply supported square, SSSS) and 7 (clamped circle) in a logarithmic scale. In these figures, the relative error is defined as $\left(\omega_{L}-\omega_{L}^{C}\right) / \omega_{L}^{C}$ where $\omega_{L}$ is the linear fundamental frequency of the specified number of DOFs and $\omega_{L}^{C}$ stands for the corresponding reference (converged) value. As it can be seen, the increase in the number of elements systematically decreases the relative errors: the convergence rates are between orders 1 and 2 . This clearly indicates the efficiency (not optimality, necessarily) of the higher-order triangular element. 

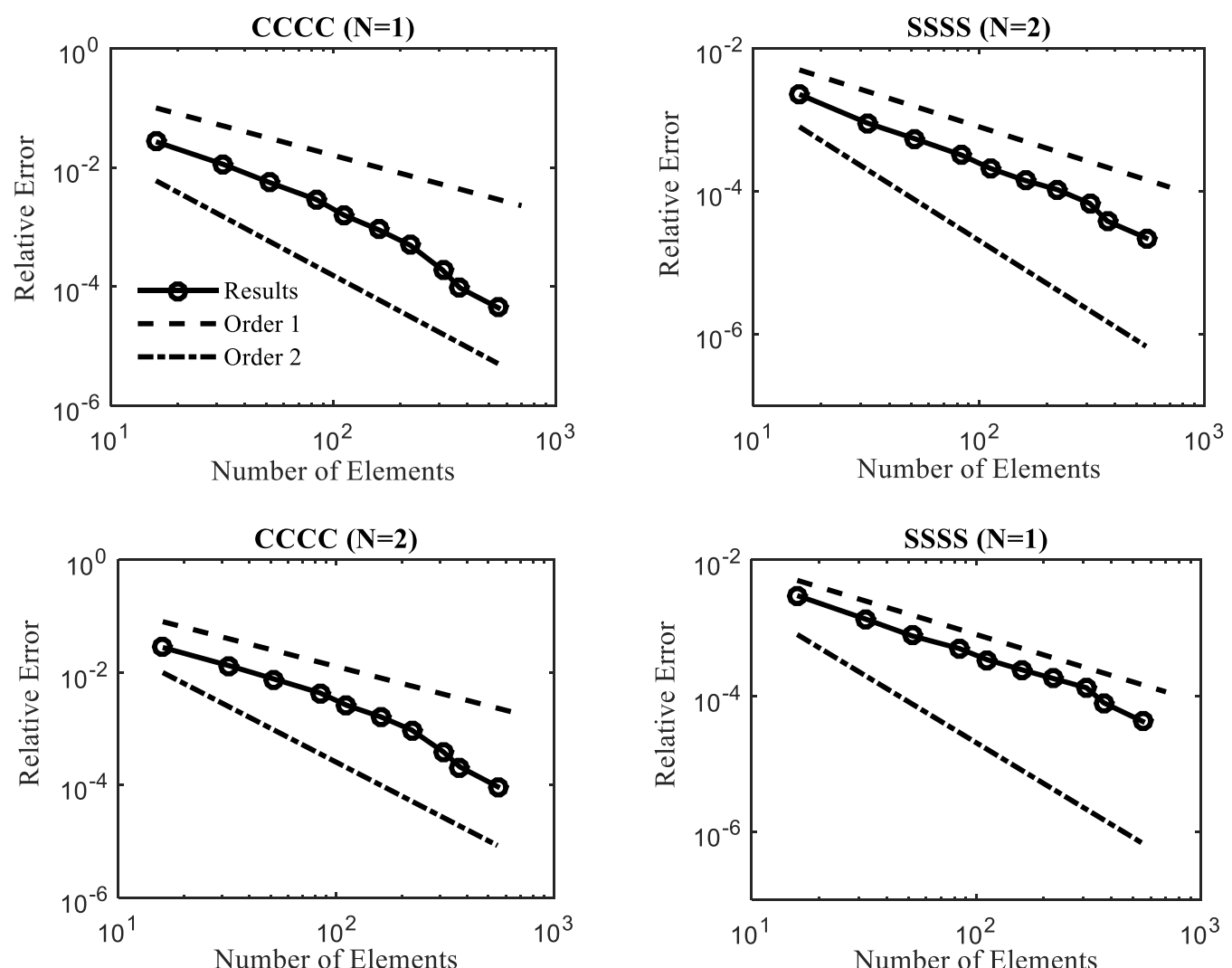

Figure 6: Convergence studies for the relative error of the linear fundamental frequency for cellular square plates $(a / h=18.48)$
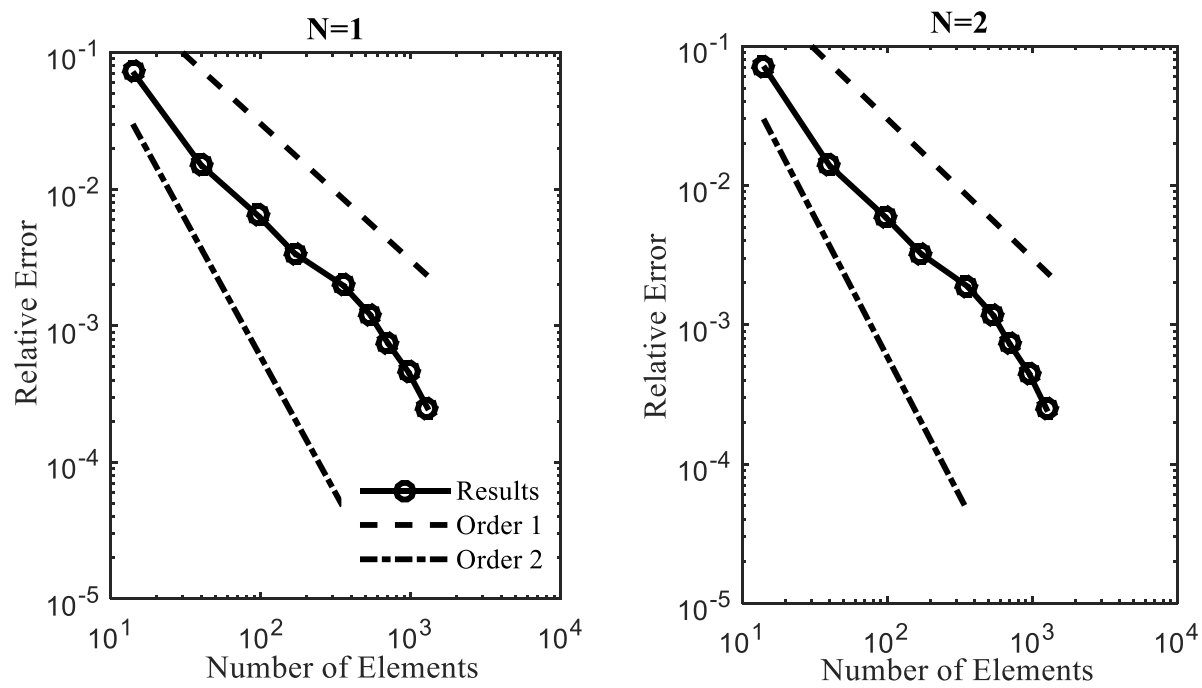

Figure 7: Convergence study for the relative error of the linear fundamental frequency of a clamped cellular circular plate $(a / h=9.24)$

Second, a set of comparisons for the first four linear frequencies of different plates versus the number of lattice layers are reported; for the shear-deformable SG plate model, the shear-deformable classical plate model and for the classical three-dimensional full-field model (serving as the true reference) are 
represented in Figures 8-11. Figures 8 and 9 are for the square plate with fully clamped and simplysupported constraints, respectively, whereas Figures 10 and 11 give the results for the plates with circular and annular mid-surfaces, respectively. The results indicate that the classical plate model cannot accurately predict natural frequencies and there are considerable differences between the classical plate and the three-dimensional full-field model. However, the SG plate model properly captures the microarchitecture-effect in the mechanical behavior of these cellular structures and there is a very good agreement between the natural frequencies predicted by the SG model and the three-dimensional model. It is worth noting that by increasing the lattice layers (say, $N>4$ ), the effects of the microarchitecture lattice diminish and the results are fairly similar for both plate models. Finally, the first four vibrational mode shapes of the example plates are illustrated in Figures 12-14 for the three-dimensional full-field model and the SG plate model. The consistency of results confirms the agreement.
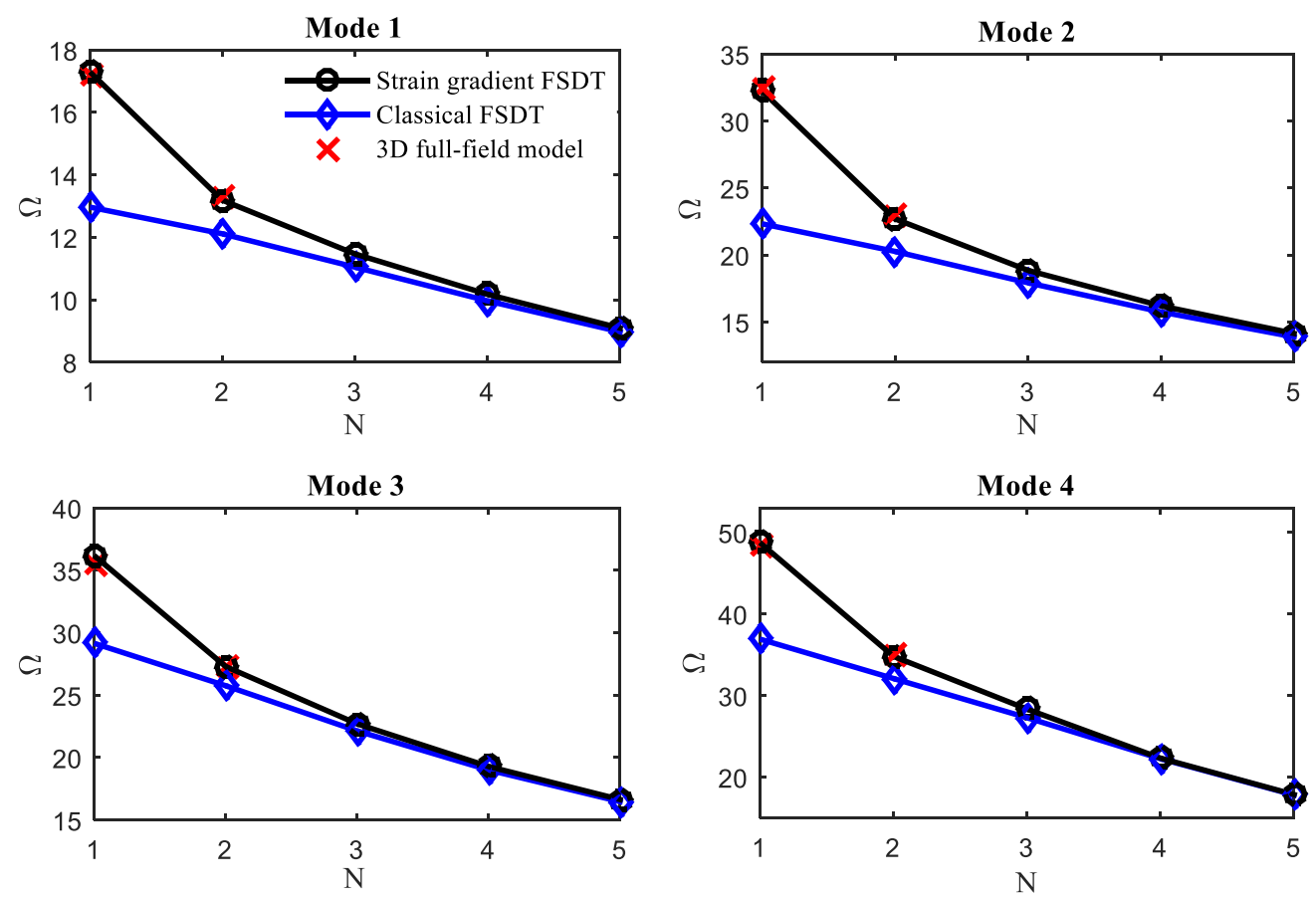

Figure 8: Comparisons for the first four linear frequencies of the square plate with different numbers $(N)$ of layers ( $a=100 \mathrm{~mm}$, clamped) 



Figure 9: Comparisons for the first four linear frequencies of the square plate with different numbers $(N)$ of layers ( $a=100 \mathrm{~mm}$, simply supported)
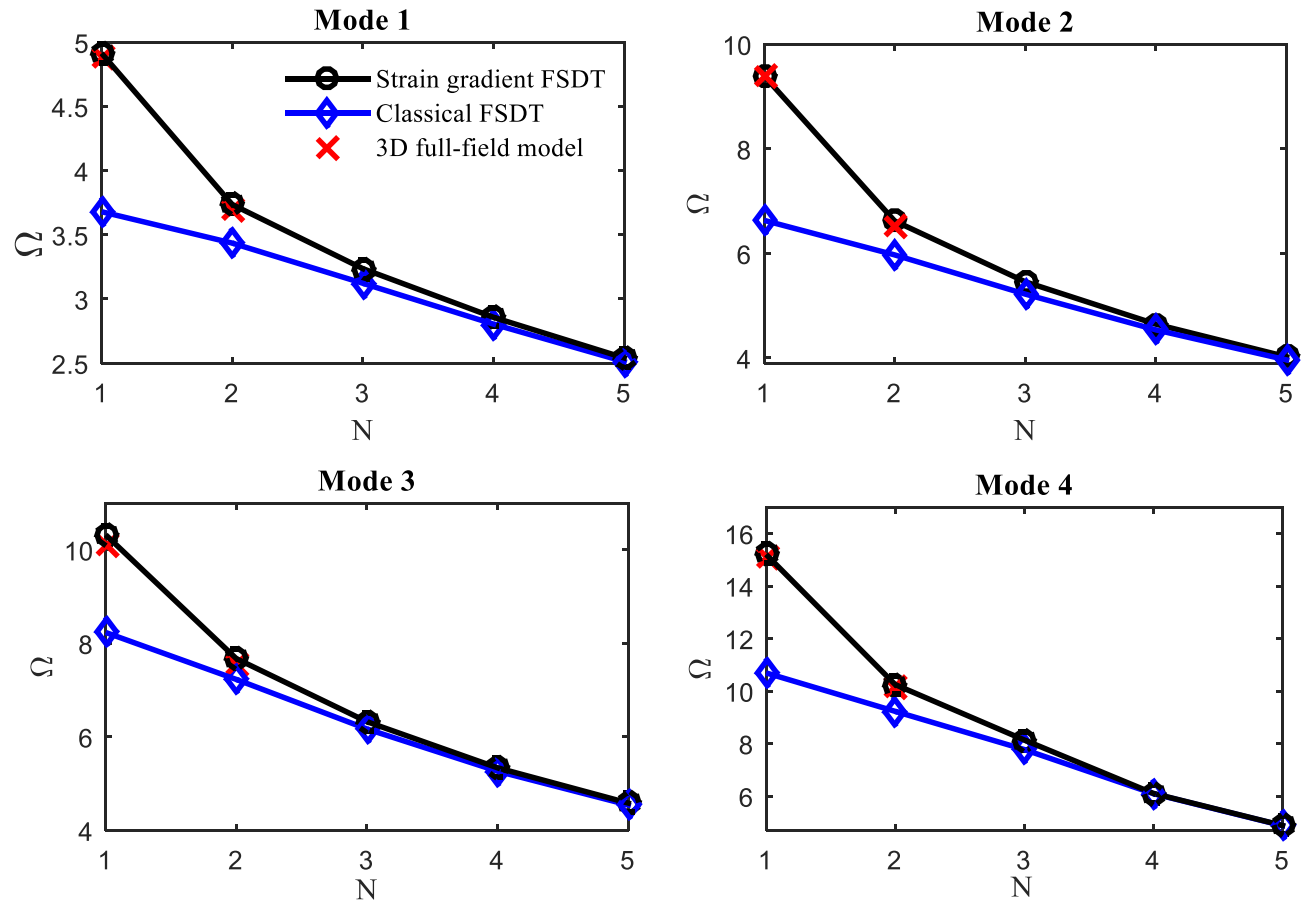

Figure 10: Comparisons for the first four linear frequencies of the circular plate with different numbers $(N)$ of layers $(a=50 \mathrm{~mm}$, clamped) 



Figure 11: Comparisons for the first four linear frequencies of the annular sector plate with different numbers $(N)$ of layers ( $a=100 \mathrm{~mm}, b=30 \mathrm{~mm}, \theta=90^{\circ}$, clamped) 
3D full-field model
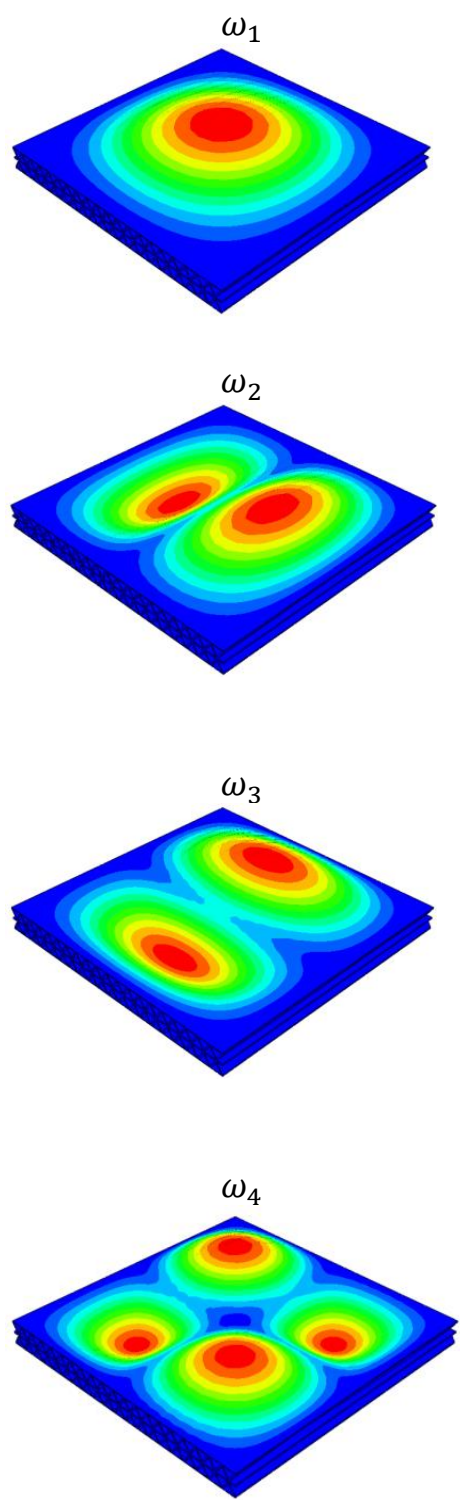

Strain gradient plate model
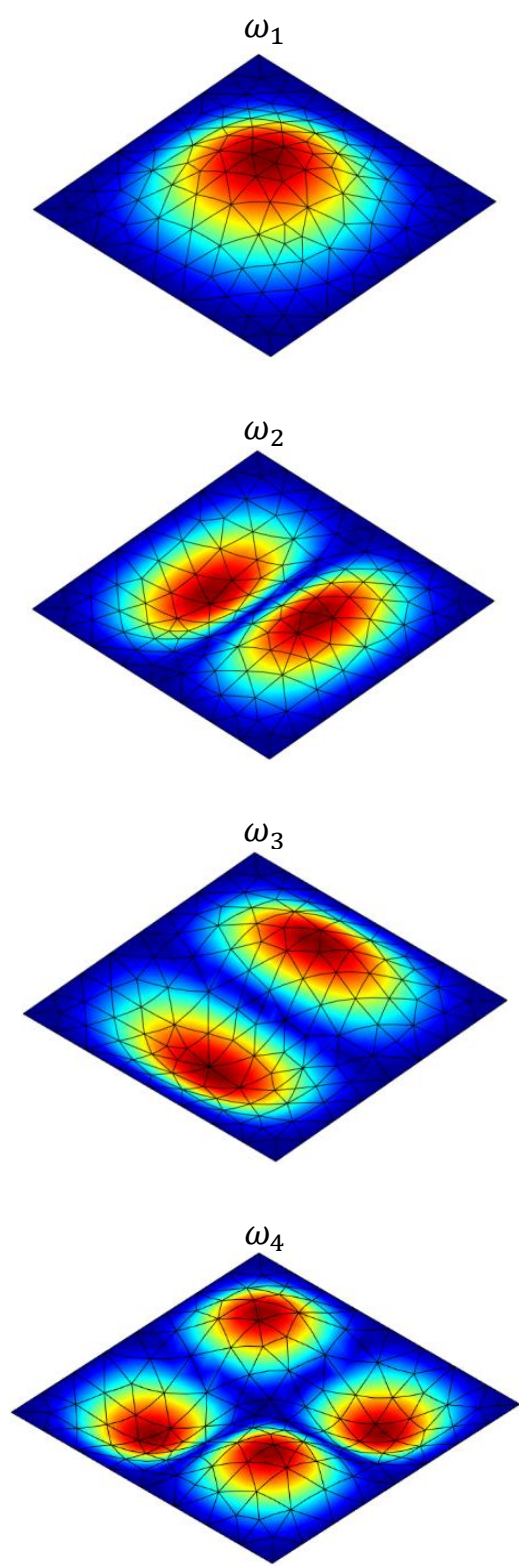

Figure 12: Comparisons for the first four mode shapes of the square plate for (left) the 3D full-field model and (right) the SG plate model ( $a=100 \mathrm{~mm}, N=2$, clamped) 
3D full-field model
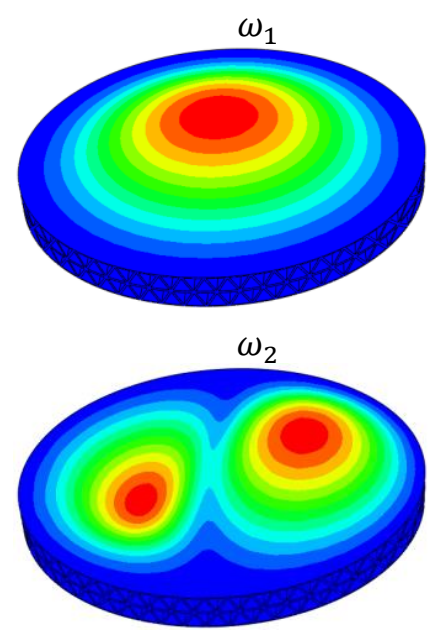

$\omega_{3}$

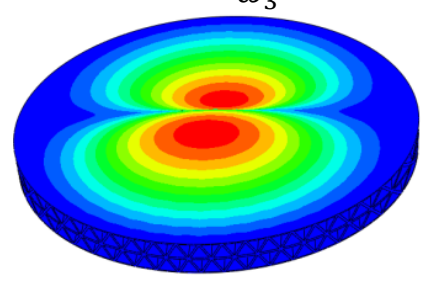

$\omega_{4}$

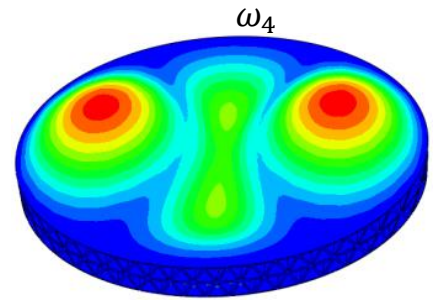

Strain gradient plate model

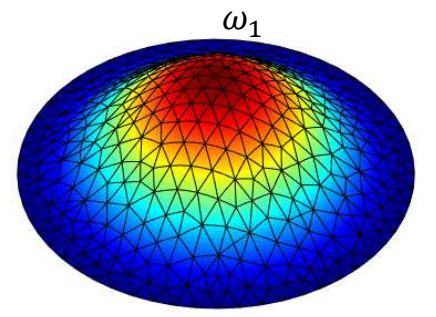

$\omega_{2}$
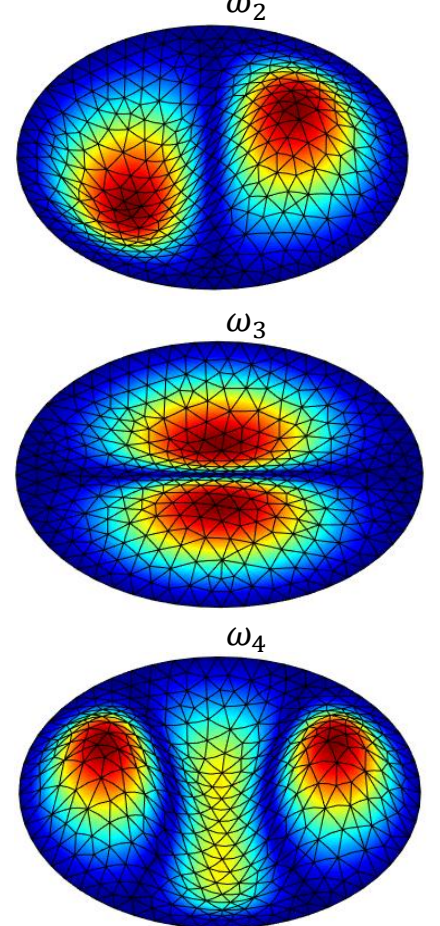

Figure 13: Comparison of the first-four mode shapes of the cellular circular plate for (left) the 3D full-field model and (right) the SG plate model ( $a=50 \mathrm{~mm}, N=2$, clamped) 
3D full-field model

$\omega_{1}$

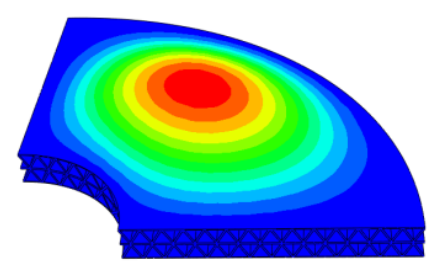

$\omega_{2}$

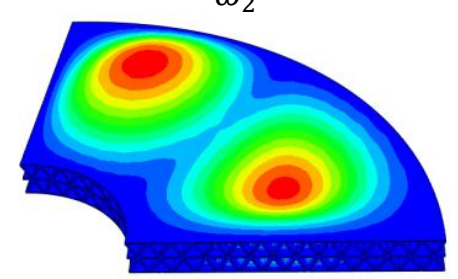

$\omega_{3}$

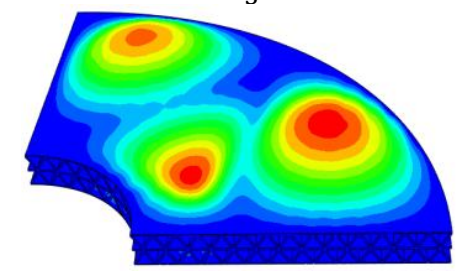

$\omega_{4}$

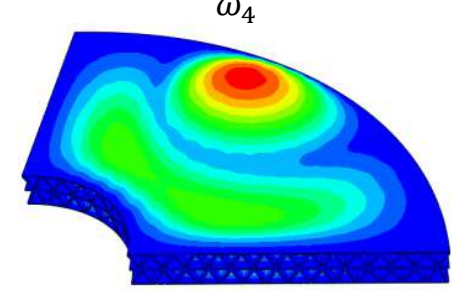

Strain gradient plate model

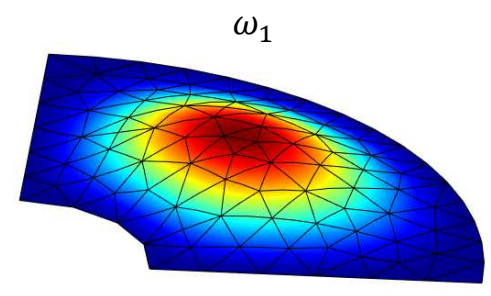

$\omega_{2}$

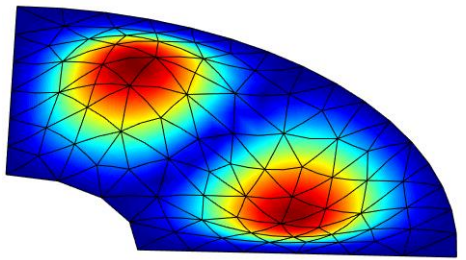

$\omega_{3}$

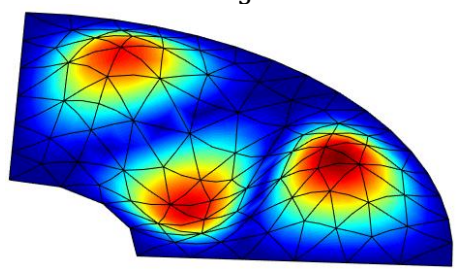

$\omega_{4}$

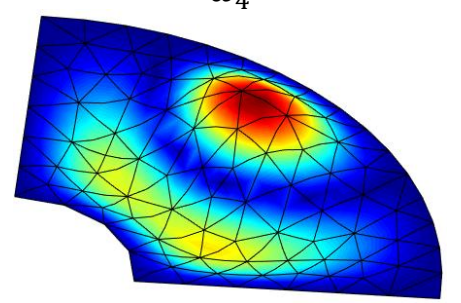

Figure 14: Comparisons for the first four mode shapes of the cellular annular sector plate for (left) the 3D fullfield model and (right) SG plate model ( $a=100 \mathrm{~mm}, b=30 \mathrm{~mm}, \theta=90^{\circ}$, clamped)

\subsection{Effects of the thickness ratio on the microarchitecture-dependent linear natural frequency}

In order to investigate the effects of thickness ratio on the vibration behavior of cellular plates, the variations of the linear frequency parameters versus the thickness ratios for different numbers of lattice layers are presented in Figures 15-17 for plates with different mid-plane shapes. The thickness ratios for different plate shapes are the following: (1) the length-to-thickness ratio for the square plates; (2) the radius-to-thickness ratio for the circular and annular sectorial plates (the outer radius for the annular plate). 

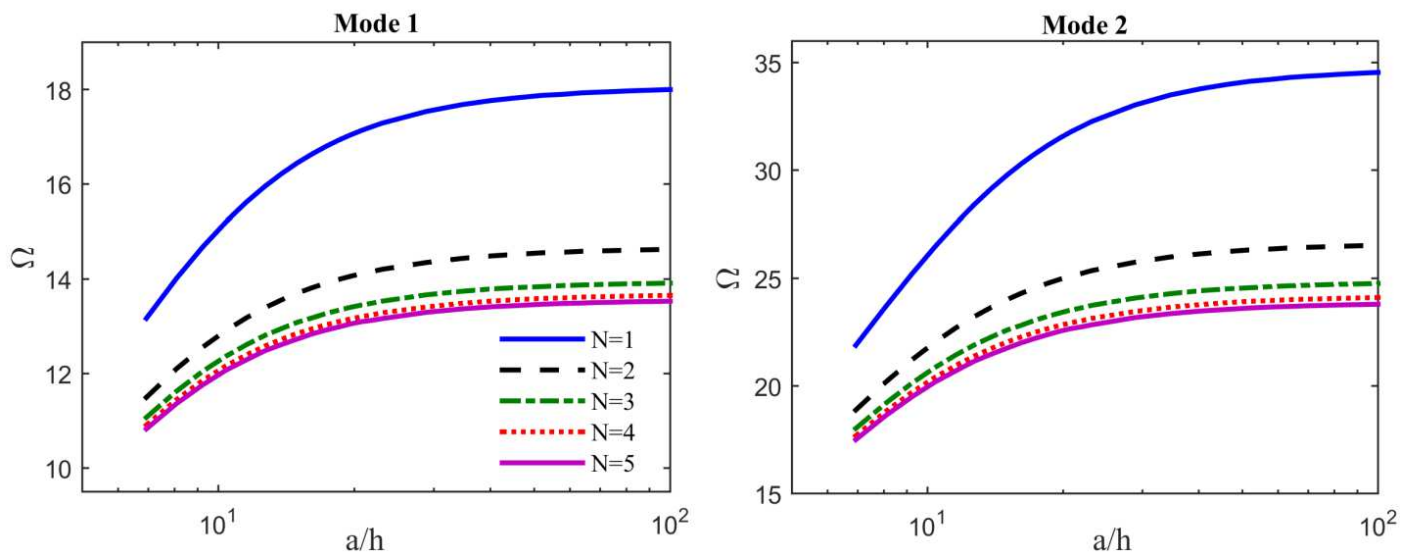

Figure 15: The impacts of the length-to-thickness ratio on the first two linear natural frequencies of the clamped square plate with different numbers $(N)$ of layers
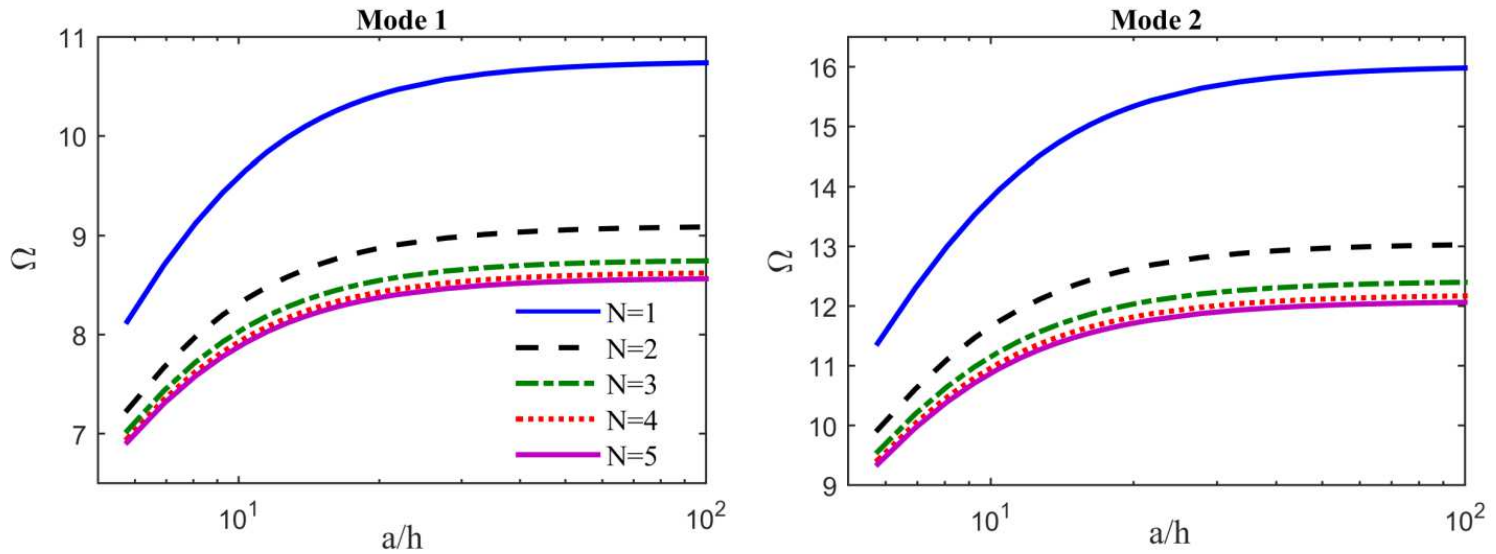

Figure 16: The impacts of the radius-to-thickness ratio on the first two linear natural frequencies of the clamped circular plate with different numbers $(N)$ of layers
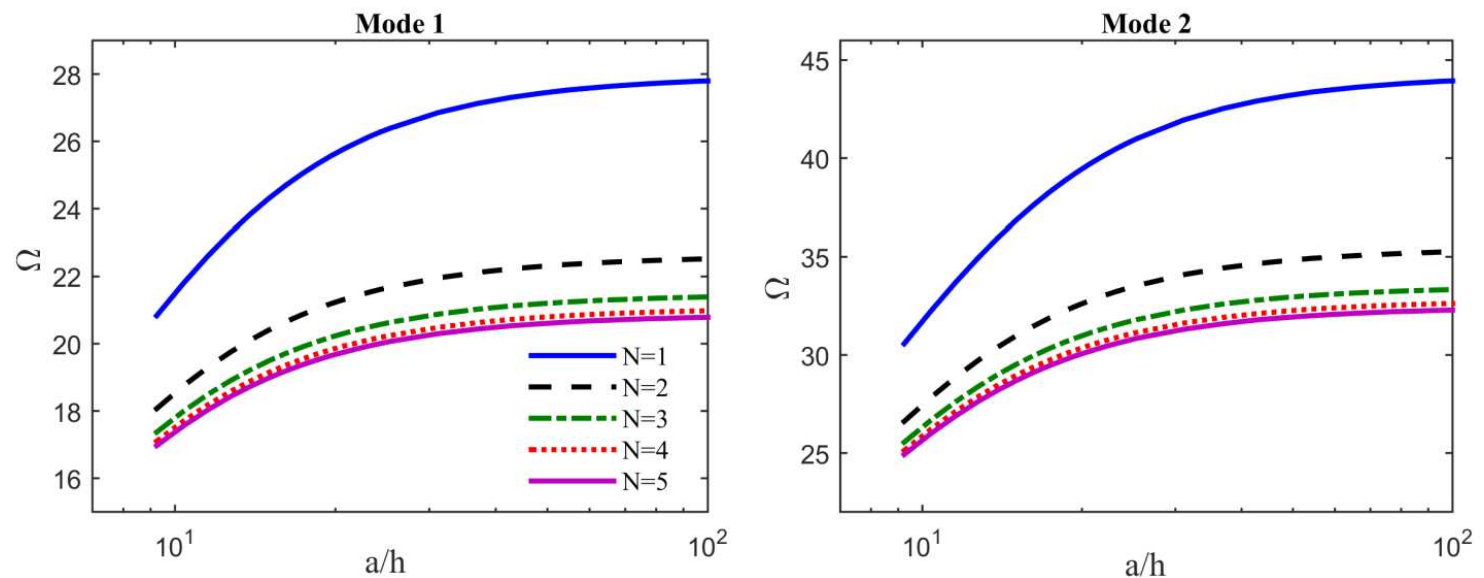

Figure 17: The impacts of the radius-to-thickness ratio on the first two linear natural frequencies of the clamped annular sector plate for different numbers $(N)$ of layers $\left(b / a=0.3, \theta=90^{\circ}\right)$ 


\subsection{Microarchitecture effects on the nonlinear free/forced vibration responses}

In this section, effects of the microarchitecture on the nonlinear free/forced vibration responses are studied. In this regard, variations of the non-dimensional deflection $\left(W_{\max } / h\right)$ versus the frequency ratio are presented in Figures 18-20 as the nonlinear vibration responses for various shapes of clamped plates. The non-dimensional force amplitude is defined as $q=f a^{4} / \mathcal{C}_{11} h^{4}$. Different numbers of lattice layers are considered with a constant thickness ratio to highlight the importance of lattice architectures. Comparing the free vibration responses for different numbers of lattice layers specifies that even with a similar thickness ratio, the one-layer plate experiences a stiffer dynamic behavior so that for the certain value of the non-dimensional deflection it has a smaller frequency ratio. Indeed, the plate with the onelayer lattice has a stiffer structure with a larger linear frequency so that it has a smaller frequency ratio. Similar dynamic behavior can be observed for the forced vibration results where the one-layer plate has a lower peak in the frequency response following its stiffer behavior owing to the lattice microarchitecture effects. The results also reveal that by the increase of the lattice layers the importance of the microarchitecture decreases and for instance the nonlinear vibration responses of three- and four-layer plates are quite similar.


Figure 18: Nonlinear free (left) and forced (right) vibration responses of the clamped cellular square plate for different number of lattice layers $(N)$ according to the SG plate theory $(a / h=18.48, q=15, c=0.02)$ 

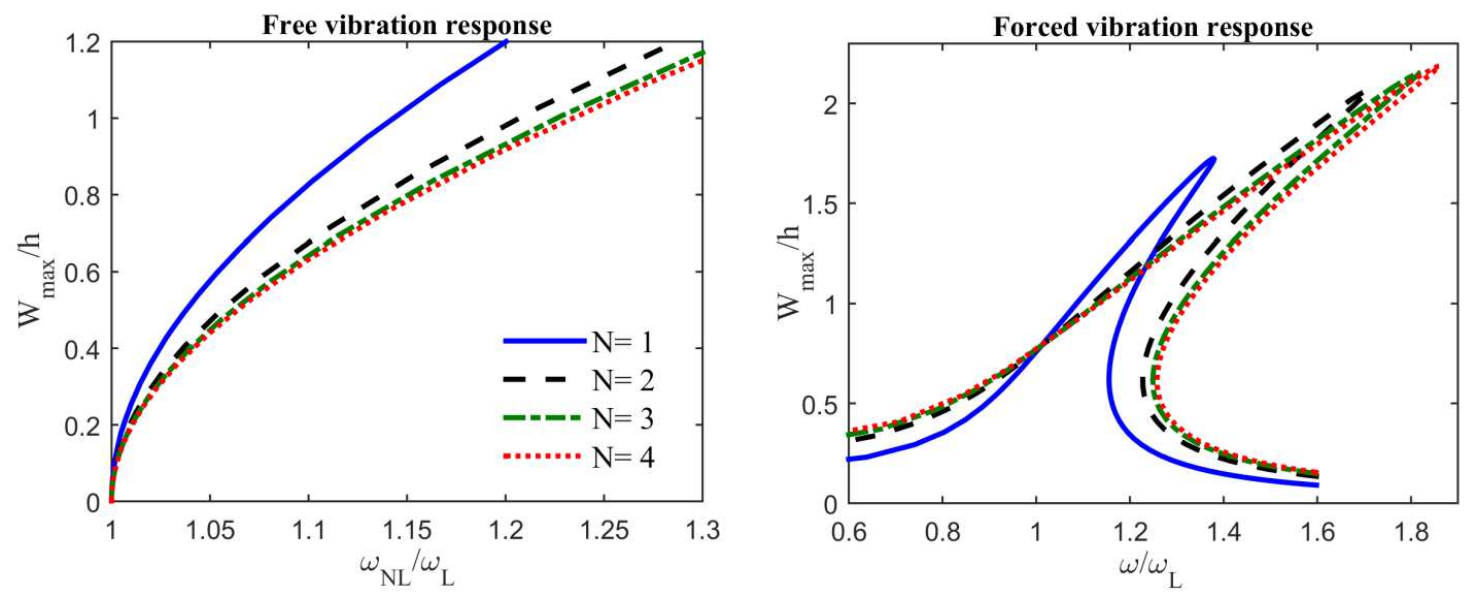

Figure 19: Nonlinear free (left) and forced (right) vibration responses of the clamped cellular circular plate with different number of lattice layers $(N)$ according to the SG plate theory $(a / h=11.55, q=2, c=0.02)$
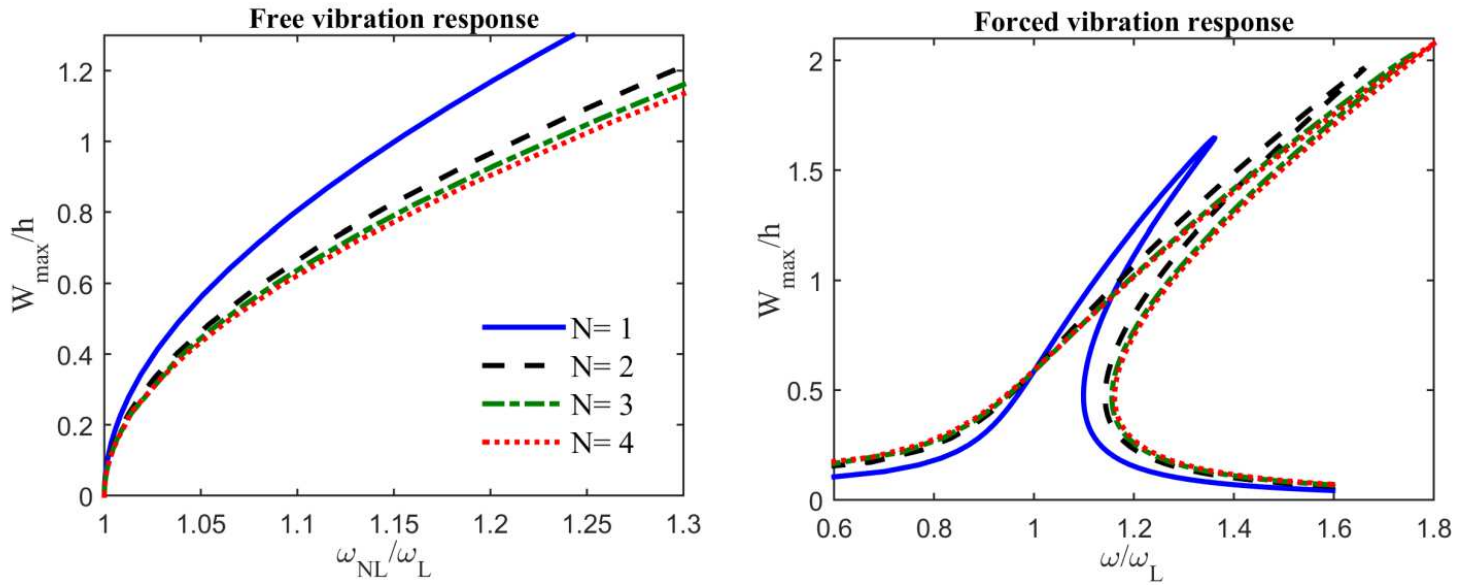

Figure 20: Nonlinear free (left) and forced (right) vibration responses of the clamped cellular annular sector plate with different number of lattice layers $(N)$ according to the SG plate theory $(a / h=18.48, b / a=0.3, q=25, c=0.015)$

\subsection{Effects of the force amplitude and damping factor on the nonlinear vibration responses}

The numerical results are represented to analyze the impacts of the force amplitude and damping coefficient on the nonlinear forced vibration responses of the cellular plates. The nonlinear frequency responses of the clamped single-layer lattice plates with square and circular mid-surface are illustrated in Figures 21 and 22 for different values of a non-dimensional force amplitude $\left(q=f a^{4} / \mathcal{C}_{11} h^{4}\right)$ and damping coefficient. It is found that larger excitation force amplitude leads to higher peaks in the nonlinear frequency response curves and makes the resonance phenomenon happen at the higher excitation frequencies. Besides, comparing the results for different damping coefficients indicates that higher values of damping coefficient result in a stiffer structure with a smaller vibration amplitude. 
(a)

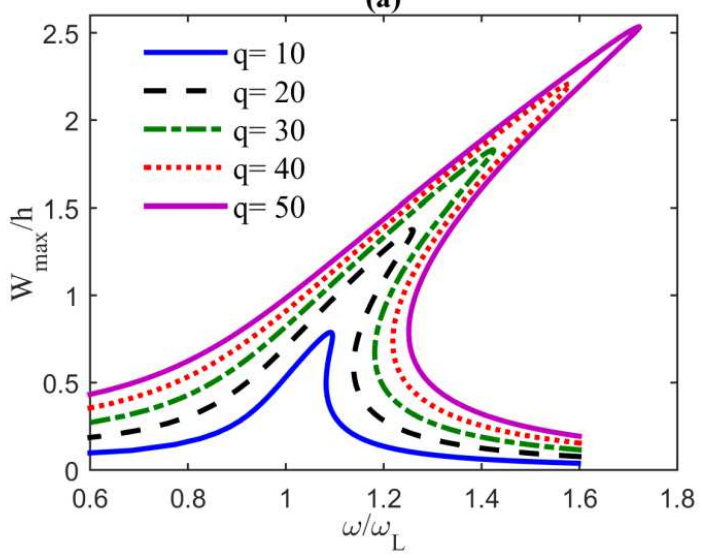

(b)

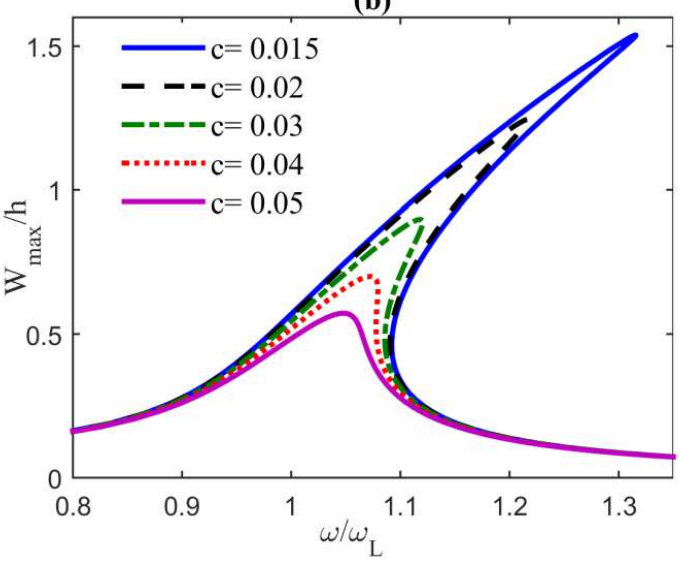

Figure 21: Effects of (a) force amplitude and (b) damping coefficient on nonlinear forced vibration responses of the cellular square plate according to the SG plate theory $(N=1, a / h=18.48$, clamped $)$

(a)

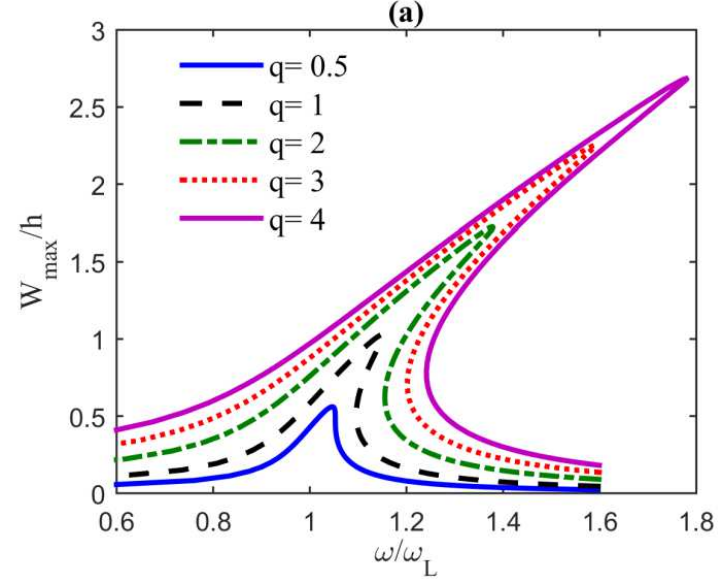

(b)



Figure 22: Effects of a) force amplitude and b) damping coefficient on nonlinear forced vibration responses of the cellular circular plate according to the SG plate theory

$(N=1, a / h=11 / 55$, clamped $)$

\section{Conclusion}

The microarchitecture-dependent large-amplitude free/forced vibrations of cellular plates with prismatic cores was studied within a strain gradient plate model discretized by finite element method. In the theoretical sections, the nonlinear equations of motion were derived by using the strain gradient elasticity theory and FSDPT along with the von Karman geometrically nonlinear kinematics. A detailed variational formulation was presented and a quasi-conforming higher-order triangular element was introduced to conduct the finite element discretization.

In the sections for numerical results, the efficiency of the finite element analysis was confirmed by convergence studies. Regarding the model, the effects of micro inertia were first then taken into account, but it was revealed that the inertial length scale parameter has no remarkable impact on the lowest natural 
frequencies investigated in the rest of the examples. For a wide range of examples of cellular plates with different shapes of mid-surface, the response of the plate model was compared to the response of the corresponding three-dimensional full-field model used as a reference, to address the accuracy of the proposed two-dimensional model. The results indicated that in the case of constant thickness ratio, the one-layer lattice plate has the stiffest mechanical behavior with higher natural frequencies due to the microarchitecture effects. It is also observed that for thin cellular plates the lattice microarchitecture has a more significant stiffening effect than for thick plates. Besides, it is found that due to the higher impact of the lattice microarchitecture, the one-layer plate has a lower peak of the forced vibration response. Comparing the results for plates with different numbers of lattice layers revealed that by the increase of the number of layers the importance of the lattice microarchitecture diminishes and the dynamic behavior can be captured by the classical shear-deformable plate theory. All in all, the results provided on the linear and nonlinear vibration of cellular plates with equitriangular lattice core, along with our previously published results for plate bending statics $[48,49]$ - to be extended towards shell structures - provide a comprehensive and reliable basis and efficient simulation tools for the structural design and engineering of microarchitectural plate structures.

Data Availability Statement: The datasets generated during and/or analyzed during the current study are available from the corresponding author on reasonable request.

Conflict of interest: The authors declare that they have no conflict of interest.

Funding: No funding was received for conducting this study.

\section{References}

1. Schaedler, T. A., \& Carter, W. B. (2016). Architected cellular materials. Annual Review of Materials Research, 46, 187-210, https://doi.org/10.1146/annurev-matsci-070115-031624.

2. Eringen, A. C. (1966). Linear theory of micropolar elasticity. Journal of Mathematics and Mechanics, 15, 909-923, http://www.jstor.org/stable/24901442

3. Mindlin, R. D. (1964). Micro-structure in linear elasticity. Archive for Rational Mechanics and Analysis, 16(1), 51-78.

4. Mindlin, R. D. (1965). Second gradient of strain and surface-tension in linear elasticity. International Journal of Solids and Structures, 1(4), 417-438, https://doi.org/10.1016/0020-7683(65)90006-5.

5. Eringen, A. C. (1972). Linear theory of nonlocal elasticity and dispersion of plane waves. International Journal of Engineering Science, 10(5), 425-435, https://doi.org/10.1016/0020$\underline{7225(72) 90050-X}$. 
6. Lam, D. C., Yang, F., Chong, A. C. M., Wang, J., \& Tong, P. (2003). Experiments and theory in strain gradient elasticity. Journal of the Mechanics and Physics of Solids, 51(8), 1477-1508, https://doi.org/10.1016/S0022-5096(03)00053-X.

7. Haque, M. A., \& Saif, M. T. A. (2003). Strain gradient effect in nanoscale thin films. Acta Materialia, 51(11), 3053-3061, https://doi.org/10.1016/S1359-6454(03)00116-2.

8. Thai, H. T., Vo, T. P., Nguyen, T. K., \& Kim, S. E. (2017). A review of continuum mechanics models for size-dependent analysis of beams and plates. Composite Structures, 177, 196-219, https://doi.org/10.1016/j.compstruct.2017.06.040.

9. Andreaus, U., Dell’Isola, F., Giorgio, I., Placidi, L., Lekszycki, T., \& Rizzi, N. L. (2016). Numerical simulations of classical problems in two-dimensional (non) linear second gradient elasticity. International Journal of Engineering Science, 108, 34-50, https://doi.org/10.1016/j.ijengsci.2016.08.003.

10. Ansari, R., \& Torabi, J. (2016). Numerical study on the free vibration of carbon nanocones resting on elastic foundation using nonlocal shell model. Applied Physics A, 122(12), 1-13, https://doi.org/10.1007/s00339-016-0602-x.

11. Awrejcewicz, J., Kudra, G., \& Mazur, O. (2021). Double mode model of size-dependent chaotic vibrations of nanoplates based on the nonlocal elasticity theory. Nonlinear Dynamics, https://doi.org/10.1007/s11071-021-06224-6.

12. Niiranen, J., \& Niemi, A. H. (2017). Variational formulations and general boundary conditions for sixth-order boundary value problems of gradient-elastic Kirchhoff plates. European Journal of Mechanics-A/Solids, 61, 164-179, https://doi.org/10.1016/j.euromechsol.2016.09.001.

13. Khakalo, S., \& Niiranen, J. (2017). Gradient-elastic stress analysis near cylindrical holes in a plane under bi-axial tension fields. International Journal of Solids and Structures, 110, 351-366, https://doi.org/10.1016/j.ijsolstr.2016.10.025.

14. Ansari, R., Torabi, J., \& Norouzzadeh, A. (2020). An integral nonlocal model for the free vibration analysis of Mindlin nanoplates using the VDQ method. The European Physical Journal Plus, 135(2), 206, https://doi.org/10.1140/epjp/s13360-019-00018-x.

15. Ghayesh, M. H., \& Farokhi, H. (2016). Parametric instability of microbeams in supercritical regime. Nonlinear Dynamics, 83(3), 1171-1183, https://doi.org/10.1007/s11071-015-2395-4.

16. Ansari, R., Oskouie, M. F., \& Rouhi, H. (2017). Studying linear and nonlinear vibrations of fractional viscoelastic Timoshenko micro-/nano-beams using the strain gradient theory. Nonlinear Dynamics, 87(1), 695-711, https://doi.org/10.1007/s11071-016-3069-6. 
17. Rouhi, H., Ebrahimi, F., Ansari, R., \& Torabi, J. (2019). Nonlinear free and forced vibration analysis of Timoshenko nanobeams based on Mindlin's second strain gradient theory. European Journal of Mechanics-A/Solids, 73, 268-281, https://doi.org/10.1016/j.euromechsol.2018.09.005.

18. Lazopoulos, K. A. (2009). On bending of strain gradient elastic micro-plates. Mechanics Research Communications, 36(7), 777-783, https://doi.org/10.1016/j.mechrescom.2009.05.005.

19. Wang, B., Zhou, S., Zhao, J., \& Chen, X. (2011). A size-dependent Kirchhoff micro-plate model based on strain gradient elasticity theory. European Journal of Mechanics-A/Solids, 30(4), 517-524, https://doi.org/10.1016/j.euromechsol.2011.04.00.

20. Ramezani, S. (2013). Nonlinear vibration analysis of micro-plates based on strain gradient elasticity theory. Nonlinear Dynamics, 73(3), 1399-1421, https://doi.org/10.1007/s11071-013-0872-1.

21. Soni, S., Jain, N. K., \& Joshi, P. V. (2019). Vibration and deflection analysis of thin cracked and submerged orthotropic plate under thermal environment using strain gradient theory. Nonlinear Dynamics, 96(2), 1575-1604, https://doi.org/10.1007/s11071-019-04872-3.

22. Gholami, R., \& Ansari, R. (2016). A most general strain gradient plate formulation for size-dependent geometrically nonlinear free vibration analysis of functionally graded shear deformable rectangular microplates. Nonlinear Dynamics, 84(4), 2403-2422, https://doi.org/10.1007/s11071-016-2653-0.

23. Balobanov, V., Kiendl, J., Khakalo, S., \& Niiranen, J. (2019). Kirchhoff-Love shells within strain gradient elasticity: weak and strong formulations and an H3-conforming isogeometric implementation. Computer Methods in Applied Mechanics and Engineering, 344, 837-857, https://doi.org/10.1016/j.cma.2018.10.006.

24. Zervos, A., Papanicolopulos, S. A., \& Vardoulakis, I. (2009). Two finite-element discretizations for gradient elasticity. Journal of engineering mechanics, 135(3), 203-213, https://doi.org/10.1061/(ASCE)0733-9399(2009)135:3(203).

25. Askes, H., Morata, I., \& Aifantis, E. C. (2008). Finite element analysis with staggered gradient elasticity. Computers \& Structures, 86(11-12), 1266-1279, https://doi.org/10.1016/j.compstruc.2007.11.002.

26. Torabi, J., Ansari, R., \& Darvizeh, M. (2018). A C1 continuous hexahedral element for nonlinear vibration analysis of nano-plates with circular cutout based on 3D strain gradient theory. Composite Structures, 205, 69-85, https://doi.org/10.1016/j.compstruct.2018.08.070.

27. Bacciocchi, M., Fantuzzi, N., \& Ferreira, A. J. M. (2020). Conforming and nonconforming laminated finite element Kirchhoff nanoplates in bending using strain gradient theory. Computers \& Structures, 239, 106322, https://doi.org/10.1016/j.compstruc.2020.106322. 
28. Ansari, R., Shojaei, M. F., Mohammadi, V., Bazdid-Vahdati, M., \& Rouhi, H. (2015). Triangular Mindlin microplate element. Computer Methods in Applied Mechanics and Engineering, 295, 56-76, https://doi.org/10.1016/j.cma.2015.06.004.

29. Torabi, J., Niiranen, J., \& Ansari, R. (2021). Nonlinear finite element analysis within strain gradient elasticity: Reissner-Mindlin plate theory versus three-dimensional theory. European Journal of Mechanics-A/Solids, 87, 104221, https://doi.org/10.1016/j.euromechsol.2021.104221.

30. Fischer, P., Klassen, M., Mergheim, J., Steinmann, P., \& Müller, R. (2011). Isogeometric analysis of 2D gradient elasticity. Computational Mechanics, 47(3), 325-334, https://doi.org/10.1007/s00466010-0543-8.

31. Niiranen, J., Khakalo, S., Balobanov, V., \& Niemi, A. H. (2016). Variational formulation and isogeometric analysis for fourth-order boundary value problems of gradient-elastic bar and plane strain/stress problems. Computer Methods in Applied Mechanics and Engineering, 308, 182-211, https://doi.org/10.1016/j.cma.2016.05.008.

32. Thai, S., Thai, H. T., Vo, T. P., \& Nguyen-Xuan, H. (2017). Nonlinear static and transient isogeometric analysis of functionally graded microplates based on the modified strain gradient theory. Engineering Structures, 153, 598-612, https://doi.org/10.1016/j.engstruct.2017.10.002.

33. Niiranen, J., Balobanov, V., Kiendl, J., \& Hosseini, S. B. (2019). Variational formulations, model comparisons and numerical methods for Euler-Bernoulli micro-and nano-beam models. Mathematics and Mechanics of Solids, 24(1), 312-335, https://doi.org/10.1177/1081286517739669.

34. dell'Isola, F., \& Steigmann, D. J. (Eds.). (2020). Discrete and Continuum Models for Complex Metamaterials. Cambridge University Press.

35. Bacigalupo, A., \& Gambarotta, L. (2019). Generalized micropolar continualization of 1D beam lattices. International Journal of Mechanical Sciences, 155, 554-570, https://doi.org/10.1016/j.ijmecsci.2019.02.018.

36. Da, D., Yvonnet, J., Xia, L., Le, M. V., \& Li, G. (2018). Topology optimization of periodic lattice structures taking into account strain gradient. Computers \& Structures, 210, 28-40, https://doi.org/10.1016/j.compstruc.2018.09.003.

37. Polyzos, D., \& Fotiadis, D. I. (2012). Derivation of Mindlin's first and second strain gradient elastic theory via simple lattice and continuum models. International Journal of Solids and Structures, 49(34), 470-480, https://doi.org/10.1016/j.ijsolstr.2011.10.021.

38. Auffray, N., Bouchet, R., \& Brechet, Y. (2010). Strain gradient elastic homogenization of bidimensional cellular media. International Journal of Solids and Structures, 47(13), 1698-1710, https://doi.org/10.1016/j.ijsolstr.2010.03.011. 
39. Yang, H., Timofeev, D., Giorgio, I., \& Müller, W. H. (2020). Effective strain gradient continuum model of metamaterials and size effects analysis. Continuum Mechanics and Thermodynamics, doi: 10.1007/s00161-020-00910-3, https://doi.org/10.1007/s00161-020-00910-3.

40. Tran, L. V., and Niiranen, J. (2020). A geometrically nonlinear Euler-Bernoulli beam model within strain gradient elasticity with isogeometric analysis and lattice structure applications. Mathematics and Mechanics of Complex Systems, 8(4), 345-371, doi: 10.2140/memocs.2020.8.345.

41. Rueger, Z., Ha, C. S., \& Lakes, R. S. (2019). Cosserat elastic lattices. Meccanica, 54(13), 1983-1999, https://doi.org/10.1007/s11012-019-00968-7.

42. Karttunen, A. T., Reddy, J. N., \& Romanoff, J. (2019). Two-scale micropolar plate model for webcore sandwich panels. International Journal of Solids and Structures, 170, 82-94, https://doi.org/10.1016/j.ijsolstr.2019.04.026.

43. Nampally, P., Karttunen, A. T., \& Reddy, J. N. (2020). Nonlinear finite element analysis of lattice core sandwich plates. International Journal of Non-Linear Mechanics, 121, 103423, https://doi.org/10.1016/j.ijnonlinmec.2020.103423.

44. Rahali, Y., Giorgio, I., Ganghoffer, J. F., \& dell'Isola, F. (2015). Homogenization à la Piola produces second gradient continuum models for linear pantographic lattices. International Journal of Engineering Science, 97, 148-172, https://doi.org/10.1016/j.ijengsci.2015.10.003.

45. Dell'Isola, F., Seppecher, P., Spagnuolo, M.,... \& Hayat, T. (2019). Advances in pantographic structures: design, manufacturing, models, experiments and image analyses. Continuum Mechanics and Thermodynamics, 31(4), 1231-1282, https://doi.org/10.1007/s00161-019-00806-x.

46. Khakalo, S., Balobanov, V., \& Niiranen, J. (2018). Modelling size-dependent bending, buckling and vibrations of $2 \mathrm{D}$ triangular lattices by strain gradient elasticity models: applications to sandwich beams and auxetics. International Journal of Engineering Science, 127, 33-52, https://doi.org/10.1016/j.ijengsci.2018.02.004.

47. Khakalo, S., \& Niiranen, J. (2019). Lattice structures as thermoelastic strain gradient metamaterials: Evidence from full-field simulations and applications to functionally step-wise-graded $\begin{array}{llll}\text { beams. Composites } \quad \text { Part } & \text { B: } & \text { Engineering, 177, } & \text { 107224, }\end{array}$ https://doi.org/10.1016/j.compositesb.2019.107224.

48. Khakalo, S., \& Niiranen, J. (2020). Anisotropic strain gradient thermoelasticity for cellular structures: Plate models, homogenization and isogeometric analysis. Journal of the Mechanics and Physics of Solids, 134, 103728, https://doi.org/10.1016/j.jmps.2019.103728.

49. Torabi, J., \& Niiranen, J. (2021). Microarchitecture-dependent nonlinear bending analysis for cellular plates with prismatic corrugated cores via an anisotropic strain gradient plate theory of first-order 
shear

https://doi.org/10.1016/j.engstruct.2021.112117.

50. Madeo, A., Neff, P., Ghiba, I. D., Placidi, L., \& Rosi, G. (2015). Wave propagation in relaxed micromorphic continua: modeling metamaterials with frequency band-gaps. Continuum Mechanics and Thermodynamics, 27(4), 551-570, https://doi.org/10.1007/s00161-013-0329-2.

51. Reda, H., Ganghoffer, J. F., \& Lakiss, H. (2017). Micropolar dissipative models for the analysis of 2D dispersive waves in periodic lattices. Journal of Sound and Vibration, 392, 325-345, https://doi.org/10.1016/j.jsv.2016.12.007.

52. Salehian, A., \& Inman, D. J. (2010). Micropolar continuous modeling and frequency response validation of a lattice structure. Journal of Vibration and Acoustics, 132(1), 011010, https://doi.org/10.1115/1.4000472.

53. Liu, F., Wang, L., Jin, D., Liu, X., \& Lu, P. (2020). Equivalent micropolar beam model for spatial vibration analysis of planar repetitive truss structure with flexible joints. International Journal of Mechanical Sciences, 165, 105202, https://doi.org/10.1016/j.ijmecsci.2019.105202.

54. Su, W., \& Liu, S. (2014). Vibration analysis of periodic cellular solids based on an effective couplestress continuum model. International Journal of Solids and Structures, 51(14), 2676-2686, https://doi.org/10.1016/j.ijsolstr.2014.03.043.

55. Challamel, N., Hache, F., Elishakoff, I., \& Wang, C. M. (2016). Buckling and vibrations of microstructured rectangular plates considering phenomenological and lattice-based nonlocal $\begin{array}{llll}\text { continuum } \quad \text { models. } & \text { Composite } & \text { Structures, } & 149,\end{array}$ https://doi.org/10.1016/j.compstruct.2016.04.007.

56. Hasrati, E., Ansari, R., \& Torabi, J. (2018). A novel numerical solution strategy for solving nonlinear free and forced vibration problems of cylindrical shells. Applied Mathematical Modelling, 53, 653672, https://doi.org/10.1016/j.apm.2017.08.027.

57. Ansari, R., Shojaei, M. F., \& Gholami, R. (2016). Size-dependent nonlinear mechanical behavior of third-order shear deformable functionally graded microbeams using the variational differential quadrature method. Composite 669-683, https://doi.org/10.1016/j.compstruct.2015.10.043. 